\title{
ITIH5 mediates epigenetic reprogramming of breast cancer cells
}

\author{
Michael Rose ${ }^{1 \dagger}$, Vera Kloten ${ }^{1 \dagger}$, Erik Noetzel ${ }^{2}$, Lukas Gola', Josef Ehling ${ }^{3}$, Timon Heide ${ }^{1}$, Steffen K. Meurer ${ }^{4}$, \\ Aljona Gaiko-Shcherbak', Antonio S. Sechi ${ }^{5}$, Sebastian Huth', Ralf Weiskirchen ${ }^{4}$, Oliver Klaas', \\ Wiebke Antonopoulos', Qiong Lin ${ }^{5,6}$, Wolfgang Wagner ${ }^{5,6}$, Jürgen Veeck ${ }^{1,7^{\wedge}}$, Felix Gremse ${ }^{3}$, Julia Steitz ${ }^{8}$, \\ Ruth Knüchel ${ }^{1}$ and Edgar Dahl ${ }^{1^{*}}$
}

\begin{abstract}
Background: Extracellular matrix (ECM) is known to maintain epithelial integrity. In carcinogenesis ECM degradation triggers metastasis by controlling migration and differentiation including cancer stem cell (CSC) characteristics. The ECM-modulator inter- a-trypsin inhibitor heavy chain family member five (ITIH5) was recently identified as tumor suppressor potentially involved in impairing breast cancer progression but molecular mechanisms underlying its function are still elusive.

Methods: ITIH5 expression was analyzed using the public TCGA portal. ITIH5-overexpressing single-cell clones were established based on T47D and MDA-MB-231 cell lines. Colony formation, growth, apoptosis, migration, matrix adhesion, traction force analyses and polarization of tumor cells were studied in vitro. Tumor-initiating characteristics were analyzed by generating a metastasis mouse model. To identify ITIH5-affected pathways we utilized genome wide gene expression and DNA methylation profiles. RNA-interference targeting the ITIH5downstream regulated gene DAPK1 was used to confirm functional involvement.

Results: ITIH5 loss was pronounced in breast cancer subtypes with unfavorable prognosis like basal-type tumors. Functionally, cell and colony formation was impaired after ITIH5 re-expression in both cell lines. In a metastasis mouse model, ITIH5 expressing MDA-MB-231 cells almost completely failed to initiate lung metastases. In these metastatic cells ITIH5 modulated cell-matrix adhesion dynamics and altered biomechanical cues. The profile of integrin receptors was shifted towards $\beta 1$-integrin accompanied by decreased Rac1 and increased RhoA activity in ITIH5-expressing clones while cell polarization and single-cell migration was impaired. Instead ITIH5 expression triggered the formation of epithelial-like cell clusters that underwent an epigenetic reprogramming. 214 promoter regions potentially marked with either H3K4 and /or H3K27 methylation showed a hyper- or hypomethylated DNA configuration due to ITIH5 expression finally leading to re-expression of the tumor suppressor DAPK1. In turn, RNAi-mediated knockdown of DAPK1 in ITIH5-expressing MDA-MB-231 single-cell clones clearly restored cell motility.

(Continued on next page)
\end{abstract}

\footnotetext{
* Correspondence: edahl@ukaachen.de

${ }^{\dagger}$ Equal contributors

Deceased

${ }^{1}$ Institute of Pathology, Medical Faculty of the RWTH Aachen University,

Aachen, Germany

Full list of author information is available at the end of the article
} 
(Continued from previous page)

Conclusions: Our results provide evidence that ITIH5 triggers a reprogramming of breast cancer cells with known stem CSC properties towards an epithelial-like phenotype through global epigenetic changes effecting known tumor suppressor genes like DAPK1. Therewith, ITIH5 may represent an ECM modulator in epithelial breast tissue mediating suppression of tumor initiating cancer cell characteristics which are thought being responsible for the metastasis of breast cancer.

Keywords: ITIH5, Breast cancer, Extracellular matrix, Epigenetic reprogramming, Cancer stem cells, DAPK1,

\section{Background}

Turnover of the extracellular matrix (ECM) is a critical step in various aspects of tumor cell biology, e.g. in orchestrating breast cancer cell differentiation driving malignancy and metastasis $[1,2]$. Inter- $\alpha$-trypsin inhibitory (ITI) proteins comprise a family of secreted serine protease inhibitors found in both the ECM and in the blood circulation [3]. ITIs are composed of a light chain, also called Bikunin, and different homologous heavy chains (i.e. ITIHs). ITIHs are covalently linked to Bikunin and thereby form a structural and functionally unique protein with a plasma protease inhibitory activity [4]. Beyond this the biological function of ITI heavy chains remains largely unknown. Trimming of precursor ITIH proteins at a conserved cleavage site unmasks a Cterminal amino acid [4], which is involved in hyaluronic acid (HA) binding [5]. Owing to that ITI heavy chains were originally referred to as serum-derived HA associated proteins (SHAPs) [6], implicating a wide spectrum of biological activities. HA that is the major proteoglycan of the ECM interacts with a large number of HAbinding proteins (HABPs) [4] like HA-receptors CD44 and RHAMM [7, 8]. Unlike all other described HABPs, ITI heavy chains are covalently linked with HA [3], whose complexation generate stable "cable-like structures" supporting ECM integrity. In 1994 Chen and colleagues showed that ITI heavy chains are involved in organizing and controlling of the cumulus-oocyte expansion [9]. In carcinogenesis of various tumor entities, accumulating studies propose a tumor suppressive role of ITI heavy chains mediated by their ECM-stabilizing activity [10-12]. ITIH1 and ITIH3, for instance, have been demonstrated to cause clear retardation of lung metastasis in vivo [12] thereby suggesting an important role of ITI heavy chains in repressing malignant diseases independently of Bikunin.

In 2004 we identified ITIH5 as the fifth heavy chain member of the ITI family [13]. ITIH5 contains all structural features found in ITIH1-3, including distinct functional domains (VIT and vWA) and the conserved cleavage site. Nevertheless, its expression pattern differs from that of other heavy chains, i.e. ITIH5 is abundantly expressed in the placenta and moderately expressed in various organs such as the mammary gland [13] indicating a local, tissue-specific function. ITIH5 dysfunction has been shown to contribute to inflammatory skin diseases [14] and obesity, thus potentially acting as regulator of human metabolism [15]. In tumor development, downregulation of ITIH5 caused by aberrant DNA hypermethylation has been reported in breast cancer [16, 17], bladder cancer [18], colon cancer [19], gastric cancer [20] and lung cancer [21]. Based on an integrated genomic and transcriptomic approach $\mathrm{Wu}$ and colleagues recently demonstrated rare somatic ITIH5 gene mutations in lung cancer whose frequency increased up to $6 \%$ in corresponding metastases [22]. Loss of ITIH5 expression in breast and bladder cancer has been associated with clinical parameters of malignant progression and metastasis $[16,18,23]$ predicting poor prognosis in both entities. These findings strengthen a putative role of ITIH5 as a tumor suppressor in various tumor types, but mechanisms of its function have not been described so far.

In the present study we give clear evidence that the ECM modulator ITIH5 is involved in controlling breast cancer cell migration and colonization in vitro and in vivo. Moreover, ITIH5 drives an epigenetic reprogramming that reverses the aggressive phenotype of basal-like MDA-MB-231 cancer cells to an epithelial-like phenotype involving re-expression of the well-known tumor suppressor gene DAPK1.

\section{Results \\ Loss of ITIH5 mRNA expression is predominant in breast tumors of the luminal B, HER2-enriched and basal-type subtype}

Previously, we identified aberrant ITIH5 promoter hypermethylation as the molecular cause for its gene inactivation in breast cancer, which was associated with unfavorable prognosis [16]. Therefore, we initially aimed to decipher ITIH5 hypermethylation and its subtype specific expression in a large dataset of The Cancer Genome Atlas (TCGA) [24, 25], in total comprising 1095 different breast cancer samples, 113 normal breast tissues and 7 distant metastases derived from primary breast tumors.

By comparison of breast cancer with healthy control samples, a prevalent loss of ITIH5 expression was found in primary breast tumors (median fold-change (FC): 18- 
fold downregulation) (Fig. 1a). In distant metastases $(n=7)$ we still observed a clear absence of ITIH5 mRNA expression (median FC: 23.5-fold downregulation). Classifying this data set by intrinsic breast cancer subtypes based on $\mathrm{Hu}$ et al. [26] we furthermore revealed a pronounced downregulation of ITIH5 mRNA in luminal B (median FC: 31.4-fold downregulation), HER2-enriched (median FC: 22.1-fold downregulation) and basal-like breast cancer (median FC: 25.7-fold downregulation) (Fig. 1b), i.e. breast cancer subtypes known to be associated with high risk for metastasis. In this data set, univariate Kaplan-Meier analyses showed that nodalnegative patients with high ITIH5 expression tend $(p=0.057)$ to have longer overall survival when compared with low ITIH5 expression (Fig. 1c). In patients lacking distant metastases at initial diagnosis high ITIH5 expression is significantly $(p<0.05)$ associated with a longer overall survival when compared with tumors showing low ITIH5 expression (Fig. 1d).

\section{ITIH5 promotes apoptosis while suppressing colony growth of breast cancer cells and mediates a morphological shift of metastatic cells in vitro}

Addressing the role of ITIH5 in breast cancer, two different in vitro tumor models were generated reflecting both the luminal and basal subtype. Upon stable transfection using a full-length ITIH5 cDNA pBK-CMV expression vector $(\Delta \mathrm{pBK}-\mathrm{ITIH} 5$ clones, also referred to as ITIH5) or the empty vector alone $(\Delta \mathrm{pBK}$-mock clones, also referred to as mock), single-cell clones were generated based on well-differentiated, luminal T47D tumor cells (Fig. 2a) and on the metastatic, basal-type MDAMB-231 breast cancer cell line (Fig. 2b).

At first, the functional impact of forced overexpression of ITIH5 on tumor colony growth was studied using 2D colony formation assays in vitro. Macroscopic analysis of grown colonies clearly visualized reduction in colony size in dependency of ITIH5 overexpression in both (T47D and MDA-MB-231) in vitro models (Fig. 2c and d). Densitometric evaluation of grown colonies significantly confirmed reduced colony growth mediated by ITIH5 expression. Colony formation was suppressed in ITIH5-expressing T47D single cell clones $(n=3)$ by $47.8 \%$ (Fig. $2 \mathrm{c}$ ) and in MDA-MB-231 $(n=4)$ by $49.0 \%$ (Fig. $2 d$ ) compared to independent mock control clones, respectively. In line XTT proliferation analyses significantly demonstrated reduced cell growth in both cell lines in dependence of ITIH5 overexpression (Fig. 2e and f). Using a caspase-3/7 apoptosis assay, we further showed increased by $92.6 \% \quad(p<0.01)$ programmed cell-death in ITIH5-expressing T47D clones

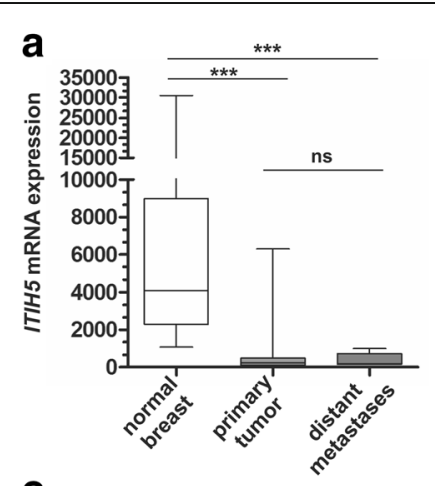

c

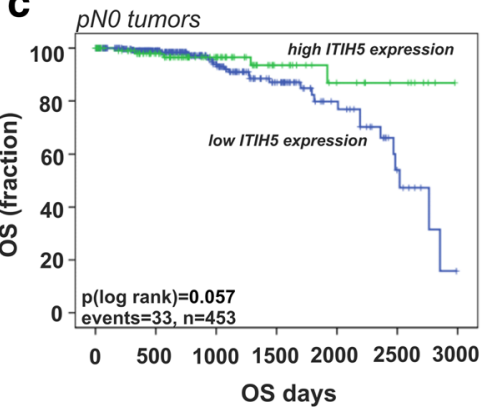

\section{b}

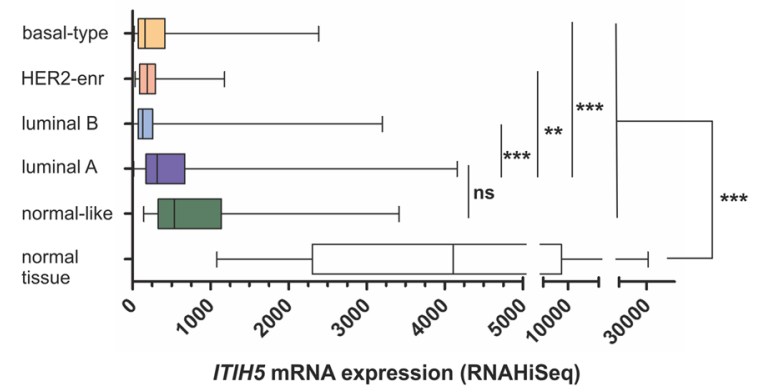

d

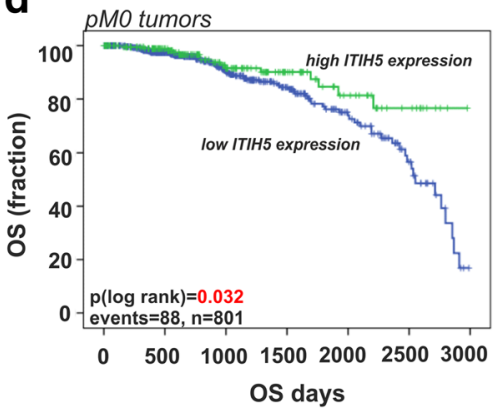

Fig. 1 ITIH5 expression loss in breast cancer subtypes and distant metastases. a-b Illustration of ITIH5 mRNA expression based on the TCGA data portal. a Box plot demonstrating a significant loss of ITIH5 mRNA expression in primary breast tumors and distant metastases derived from primary breast tumors, ns: not significant, ${ }^{* *} p<0.001$. b Breast tumor samples stratified by subtypes [28], ns: not significant, ${ }^{* *} p<0.01$; ${ }_{* * *}^{*} p<0.001$. c-d Kaplan-Meier analyses display OS of nodal-negative (pNO, c) and non-metastatic (pMO, d) breast cancer patients stratified by high ITIH5 (green curve) and low/intermediate ITIH5 mRNA expression (blue curve) 

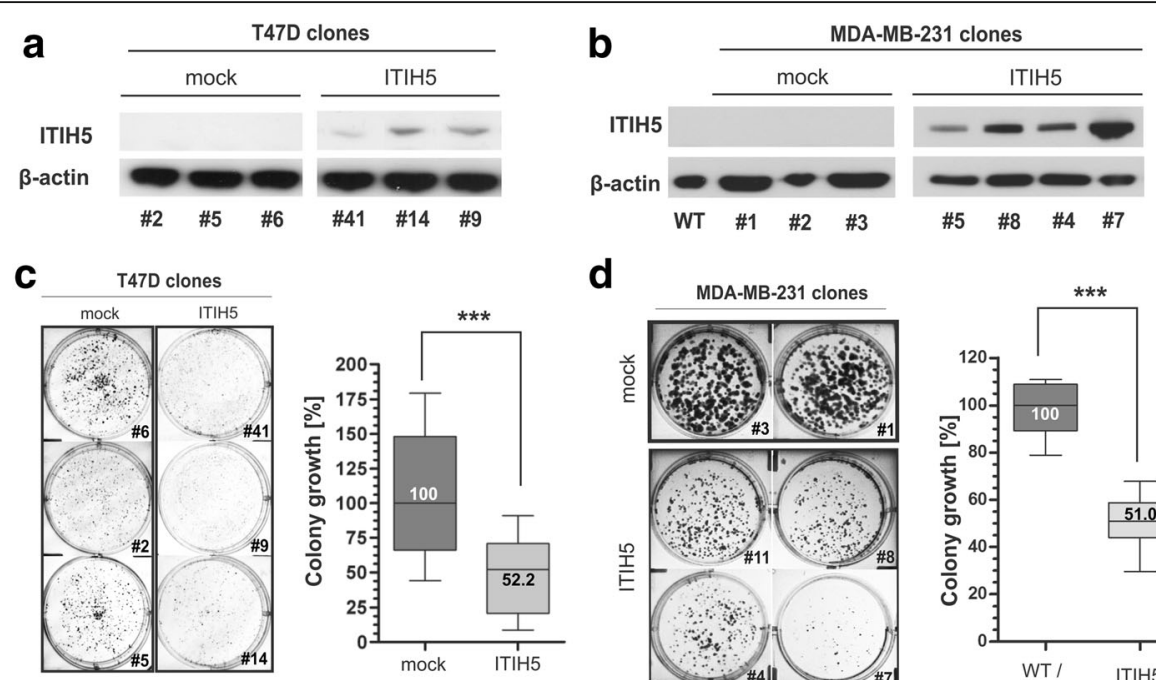

d
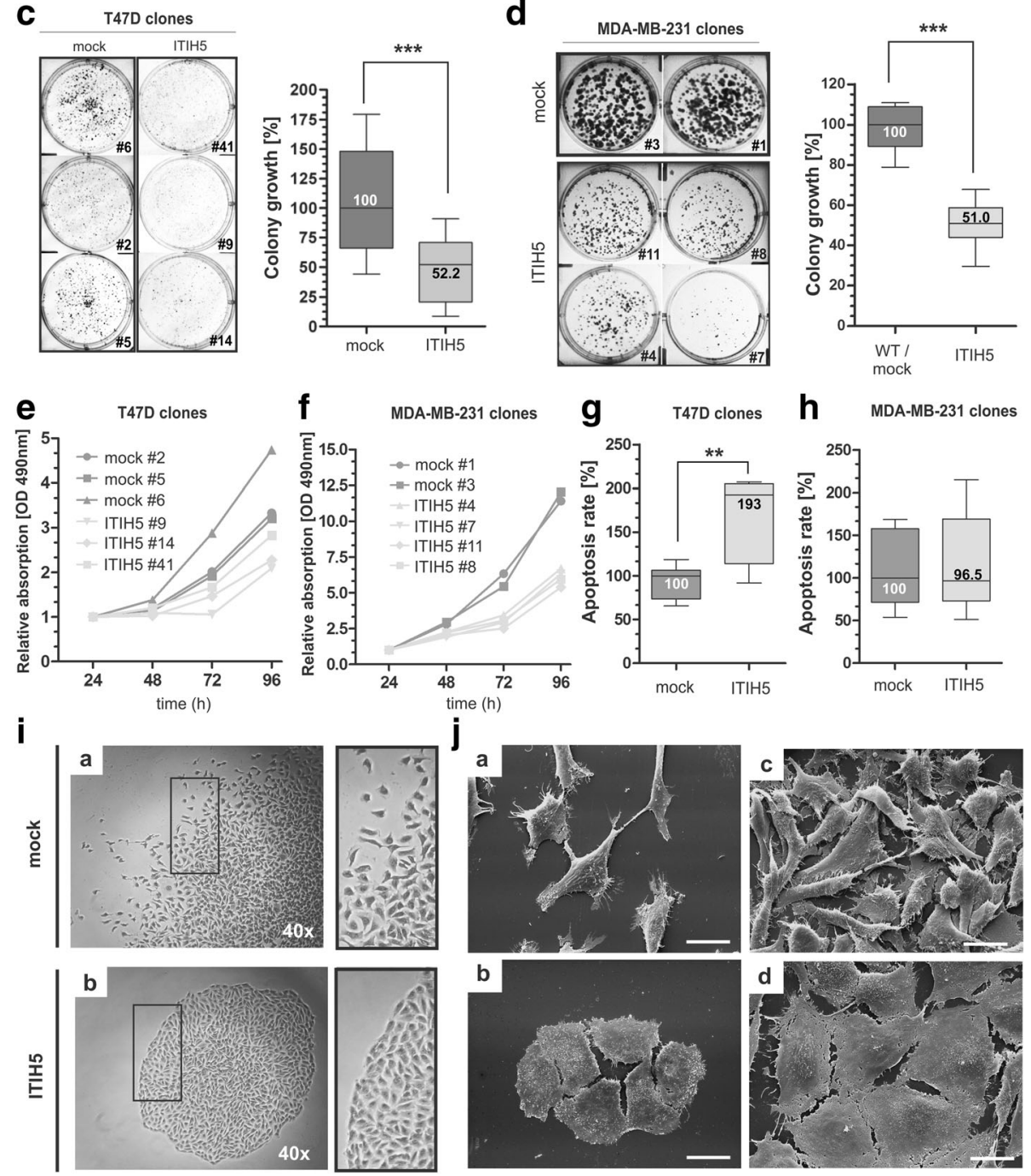

Fig. 2 ITIH5 impairs cell growth and colonization of breast cancer cells and induce a phenotype shift in vitro. a ITIH5 gain-of-function model of luminal breast cancer cells: Ectopic ITIH5 expression in transfected T47D $\triangle \mathrm{pBK}$-ITIH5 clones was confirmed by Western blotting. A specific signal of the ectopic ITIH5 protein is detectable only in T47D ITIH5 clones. $\beta$-actin served as loading control. b ITIH5 gain-of-function model of basaltype breast cancer cells: Ectopic ITIH5 expression in transfected MDA-MD-231 $\triangle \mathrm{pBK}-\mathrm{TTIH} 5$ single-cell clones was confirmed by Western blotting. A specific signal of the ectopic ITIH5 protein is detectable in MDA-MB-231 ITIH5 clones. $\beta$-actin served as loading control. c Colony growth of luminal T47D breast cancer cells in dependency of ITIH5 re-expression. Box plot presents averages of triplicate experiments based on three independent T47D ITIH5 and three T47D mock clones. Left: Representative wells with grown ITIH5 as well as mock colonies are shown. Right: Densitometrical evaluation of colony growth after 14 days. $\mathbf{d}$ Colony growth of basal-type MDA-MB-231 breast cancer cells due to stable ITIH5 re-expression. Box plot presents averages of triplicate experiments based on four independent MDA-MB-231 ITIH5 and two MDA-MB-231 mock clones. Left: Representative wells with grown $\triangle \mathrm{pBK}-\mathrm{ITIH} 5$ as well as mock colonies are shown. Right: Densitometrical evaluation of colony growth after 14 days. e-f XTT proliferation assay was performed. T47D e and MDA-MB-231 f $|T| H 5$ single-cell clones showed reduced cell growth compared with $\triangle \mathrm{pBK}$-mock controls. The baseline level at $24 \mathrm{~h}$ was set to $1 . \mathbf{g}$ - $\mathbf{h}$ Caspase $3 / 7$ activity as indicator of apoptosis in independent T47D $\mathbf{g}$ and MDAMB-231 $\mathbf{h}$ mock and ITIH5 single-cell clones $(n=3$, respectively). Box plot demonstrates relative apoptosis rate. Horizontal lines: grouped medians. Boxes: $25-75 \%$ quartiles. Vertical lines: range, minimum and maximum, ${ }^{* *} p<0.01$. i Comparison of morphological MDA-MB-231 colony growth patterns of ITIH5 and mock clones. Right images: colony edges. Representative light-micrographs are shown. $\mathbf{j}$ Comparison of single-cell plasticity showing different confluence of both MDA-MB-231 ITIH5 and mock clones. Representative SEM-micrographs are shown. Scale bar $=20 \mu \mathrm{m}$ 
( $n=3$ independent clones) compared to mock control cells ( $n=3$ independent clones) (Fig. 2g). ITIH5 expression had no sustained effect on apoptosis in MDA-MB-231 cells (Fig. 2h). In turn, microscopic analyses revealed fundamental changes in growth patterns of MDA-MB-231 $\triangle$ pBK-ITIH5 cancer cells (Fig. 2i and j) but not in T47D transfected cells (data not shown). While mock-transfected MDA-MB-231 cells retained a scattered colony growth, ITIH5-expressing MDA-MB-231 cells formed independently of the amount of forced ITIH5 expression of tested clones $(n=6$; Additional file 1) tightly packed colony structures lacking cell spreading at the colony periphery (Fig. 2i). Scanning electron microscopy analyses (Fig. 2j) finally confirmed pronounced morphological changes of independent MDAMB-231 ITIH5 cell clones at high and low density. $\Delta$ pBK-mock cells showed a mesenchymal-like morphology characterized by an elongated cell shape. In contrast, ITIH5-expressing MDA-MB-231 cells grew in a monolayer with a cuboidal single-cell shape indicating a profound impact of ITIH5 action in this metastatic breast cancer cell line.

\section{ITIH5 suppresses lung colonization by metastatic MDA-MB-231 breast cancer cells in mice}

To study the putative tumor suppressive function of ITIH5 under physiological conditions, an experimental in vivo metastasis assay was performed using single-cell clones of the highly metastatic MDA-MB-231 cell line. By day 50 after tumor cell injection (i.v.) mice were three-dimensionally (3D) screened using whole-body non-invasive $\mu \mathrm{CT}$ scans to evaluate major organs of metastatic tumor growth (Fig. 3a). No metastases were found in brain or liver, whereas lungs of control mice (injected with MDA-MB-231 $\triangle \mathrm{pBK}$-mock single-cell clones) presented high numbers of macro-metastases, i.e. up to 9 metastases/mouse (Fig. $3 \mathrm{~b}$ and c). Overall 6 out of $7(85 \%)$ mice treated with MDA-MB-231 mock cells showed lung metastases. In comparison to that, the number of macro-nodules in the lung was clearly decreased when mice received MDA-MB-231 tumor cells expressing ITIH5 as confirmed by RT-PCR (Fig. 3d). Only in 3 out of 7 (43\%) mice macro-metastases were detected. The highest number of metastases was 2 nodules per mouse (Fig. 3b). Based on histopathological assessment of lung sections, we confirmed a clear reduction of macro-metastases by ITIH5 $(p<0.05)$ (Fig. 3e and f). More interestingly, remarkable differences in the number of micrometastases $(<0.1 \mathrm{~cm})$ were found between the ITIH5group and control mice (Fig. 3f). While control mice exhibited high numbers of micro-metastases (median number: 33.25) that spread over the whole lung tissue, experimental mice receiving ITIH5-expressing tumor cells only presented very low numbers (median number: 0.5 ) of small tumor nodules.

\section{ITIH5 remodels ECM composition, enhances cell-matrix adhesion and contractile cell force generation}

So far, data on biological processes and pathways affected by ITI heavy chains besides HA-stabilization are still lacking. Therefore, a transcriptomic micro-array profiling approach was performed followed by gene ontology (GO) annotations using a gene set comparison analysis. Interestingly, over-represented gene annotations confirmed an impact of ITIH5 expression on biological processes (BP) such as "lipid catabolic process" in this metastatic MDA-MB-231 cancer cell line (Table 1) that is in line with recently published data [15]. In addition to those annotations, we further revealed an association of ITIH5 expression with categories like "cell adhesion" or "epithelial cell differentiation". Interestingly, enrichment of collagens was also shown including upregulation of both transcripts (COL4A1, FC: 1.73 and COL4A2, FC: 1.53) of the basement membrane (BM) constituent collagen type IV (Table 1). We confirmed increased expression of collagen type IV on mRNA and on protein level in MDA-MB-231 $\triangle$ pBK-ITIH5 cells (Fig. 4a and b).

Experimentally, MDA-MB-231 $\Delta$ pBK-ITIH5 clones showed altered cell-matrix adhesion dynamics in vitro. On both substrates, i.e. on Matrige ${ }^{\mathrm{Tm}}$ that imitates the BM and on HA, ITIH5 expression led to an increased cell-matrix adhesion (Matrigel ${ }^{\mathrm{Tm}}$ : $+52.6 \%, \quad p<0.001 ; \mathrm{HA}:+37.4 \%$; $p<0.001$ ) compared to mock control clones (Fig. 4c). Based on this result, cellular traction forces was investigated as potential trigger that could contribute to the modulation of cell behavior, such as enhanced matrix adhesion $[27,28]$. For this purpose traction force microscopy (TFM) was used as standard method to quantify contractile forces that cells exert on their surrounding ECM [29, 30]. In order to recapitulate a tumor relevant microenvironment, substrates of $15 \mathrm{kPa}$ stiffness were used for cell adhesion. Such ECM compliance lies in the range of activated breast tumor stroma [31], which results from continuous ECM-stiffening during cancer progression driving invasion and tissue tropism of metastatic tumor cells [32]. In vitro traction force analyses revealed strengthened contractile cell force generation during cell-matrix adhesion of ITIH5 expressing cells (Fig. 4d). The direct comparison with corresponding mock control clones showed a median increase in cell force of $43.9 \%$ in MDAMB-231 ITIH5 clones ( $\Delta$ pBK-mock: $107.5 \mathrm{nN}, \Delta \mathrm{pBK}$ ITIH5: $162.6 \mathrm{nN} ; p<0.0001$ ) (Fig. 4e).

ITIH5 modulates integrin signaling that is associated with inhibition of mesenchymal single-cell migration in vitro Next, we aimed to decipher the dynamics of the observed mechanical alterations in ECM-cell interactions 


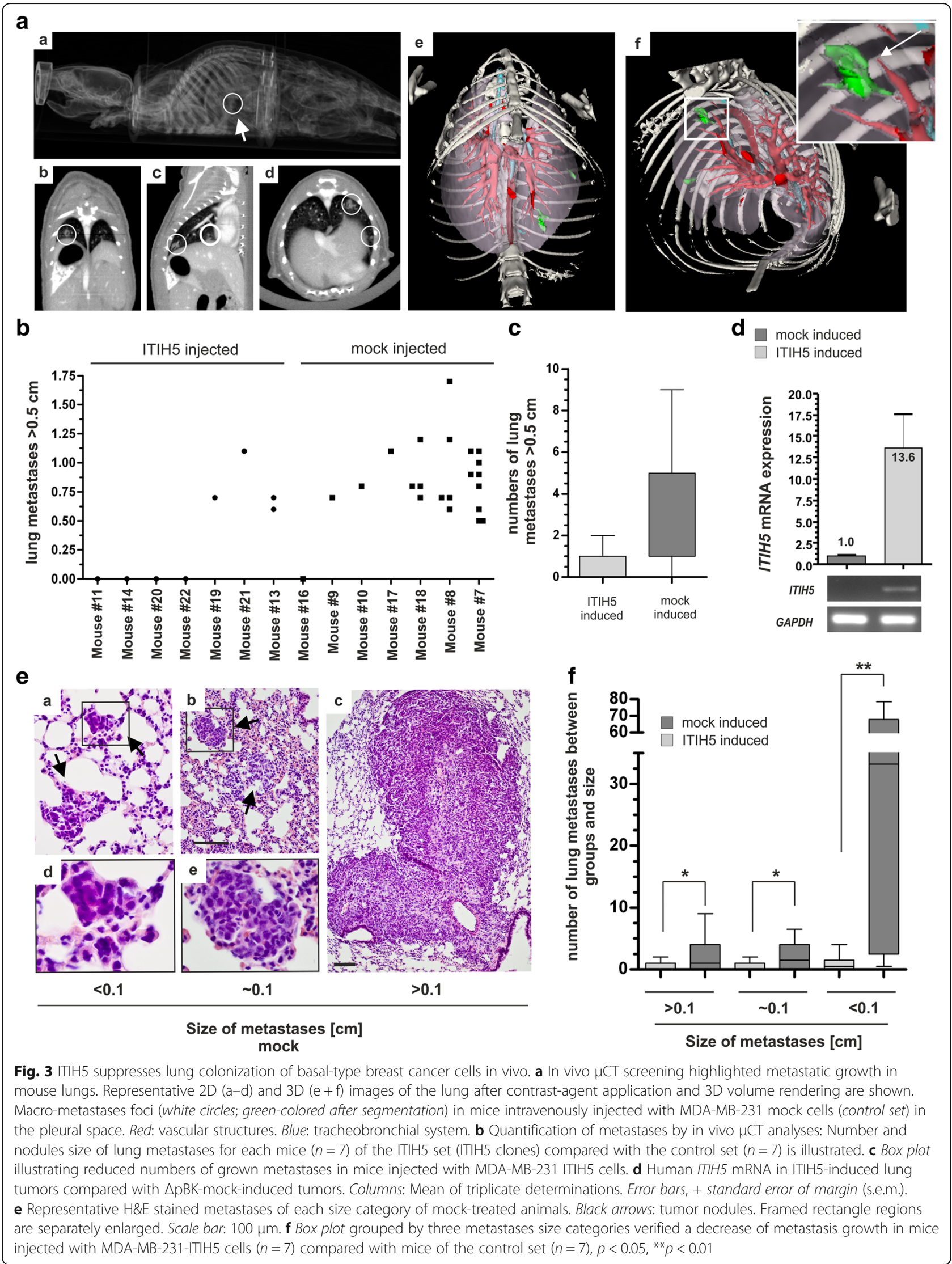


Table $1 \mathrm{GO}$ annotated biological processes and cellular components

\begin{tabular}{llllcc}
\hline GO category & GO ontology & GO term & Number of genes & LS permutation p-value & KS permutation $p$-value \\
\hline GO:0016337 & BP & cell-cell adhesion & 95 & $\mathbf{0 . 0 0 0 0 8}$ & $\mathbf{0 . 0 0 0 0 1}$ \\
GO:0016042 & BP & lipid catabolic process & 56 & $\mathbf{0 . 0 0 0 2 2}$ & $\mathbf{0 . 0 0 2 3 1}$ \\
GO:0016339 & BP & calcium-dependent cell-cell adhesion & 9 & $\mathbf{0 . 0 0 2 2 1}$ & $\mathbf{0 . 0 0 0 2}$ \\
GO:0030855 & BP & epithelial cell differentiation & 72 & $\mathbf{0 . 0 0 5 6 1}$ & $\mathbf{0 . 0 0 3 3 7}$ \\
GO:0030426 & CC & growth cone & 23 & $\mathbf{0 . 0 0 0 3 7}$ & $\mathbf{0 . 0 0 2 1 9}$ \\
GO:0030427 & CC & site of polarized growth & 24 & $\mathbf{0 . 0 0 0 5 3}$ & $\mathbf{0 . 0 0 3 2}$ \\
GO:0005578 & CC & proteinaceous extracellular matrix & 86 & $\mathbf{0 . 0 0 2 6 6}$ & $\mathbf{0 . 0 0 1 3 4}$ \\
GO:0005581 & CC & collagen & 13 & $\mathbf{0 . 0 1 6 4 4}$ & $\mathbf{0 . 0 0 3 3 2}$ \\
\hline
\end{tabular}

Bold $p$-values: significant $(p \leq 0.05)$
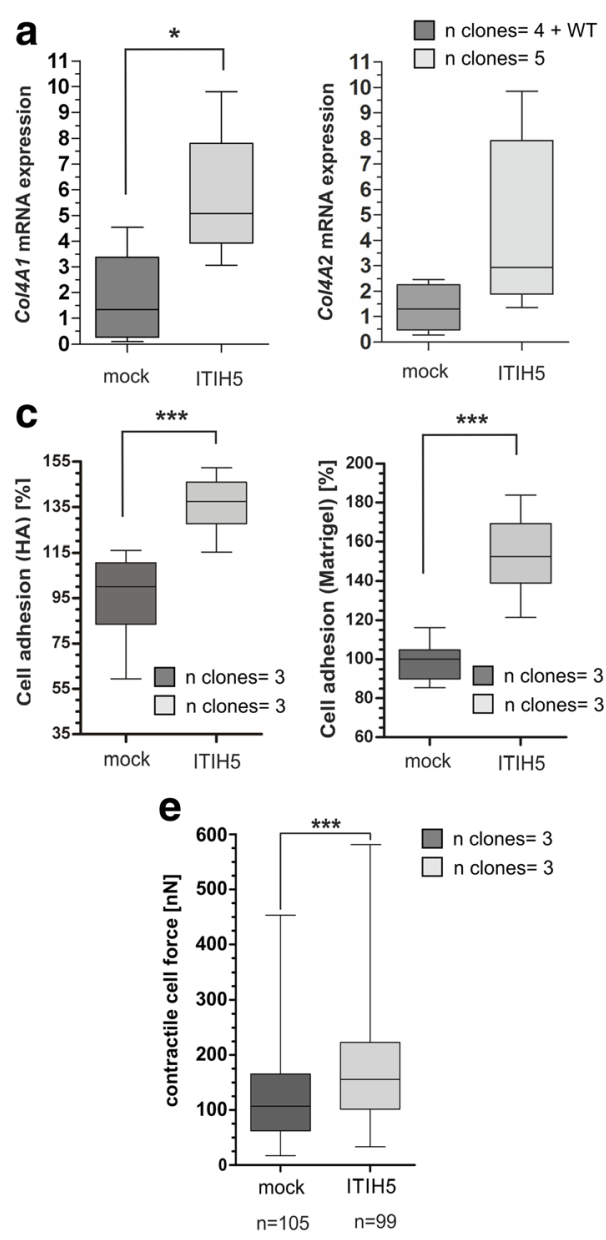

$\mathrm{n}$ clones $=3$

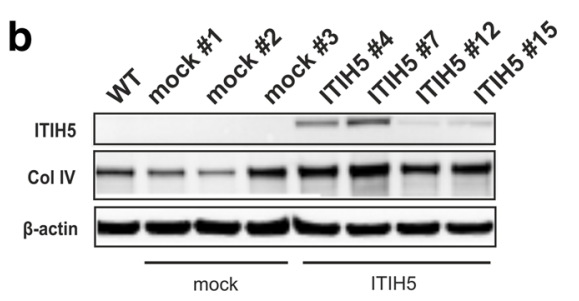

d
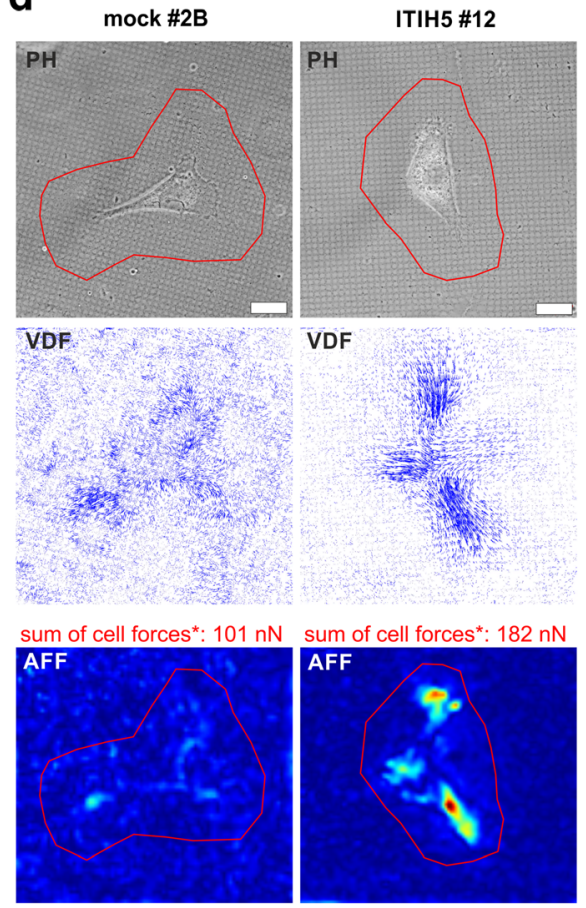

$\begin{array}{lllll}0.1 & 0.2 & 0.3 & 0.4 & 0.5\end{array}$

Fig. $4 \mathrm{ITIH} 5$ alters ECM-cell interactions and enhances cell-matrix adhesion and contractile cell force generation. $\mathbf{a}$-b Collagen type IV mRNA and protein (western blot) expression in ITIH5 and mock clones. $\beta$-actin served as loading control. ${ }^{*} p<0.05$. c Cell-to-matrix adhesion of ITIH5 and mock clones MDA-MB-231 clones on Matrige ${ }^{T \mathrm{M}}$ and HA-substrate. ${ }^{* * *} p<0.001$. d Cell traction force microscopy assay was used to measure contractile forces that cells exert on elastomeric substrate during cell-matrix adhesion. Representative phase contrast images ( $\mathrm{PH}$, upper row) of a mock clone and an ITIH5 clone adhered onto elastomeric substrates (15 kPa stiffness) are shown. Vector deformation field images (VDF, middle row) were retrieved from fluorescent nanobead displacement tracking Scale bar: $20 \mu \mathrm{m}$. Corresponding area force fields (AFF: lower row) represent the actual contractile cell force distribution per surface unit $\left(\mathrm{nN} / \mu^{2}{ }^{2}\right)$. Red ROls: Cell outlines were defined to summarize and compare the whole contractile force exerted by one single cell [nN], * : cell forces of illustrated cells. e Box plots analysis illustrate the overall comparison of contractile cell force generation of all measured mock clones (1, 2B and \#3), and ITIH5-clones (4, 7 and \#12). Box plots: Horizontal lines: grouped medians. Boxes: $25-75 \%$ quartiles. Vertical lines: range, peak and minimum; ${ }^{* * *} p<0.0001$ 
which just reflects a snapshot of the cell state so far. We focused on integrins that are known to bind ECM, especially, BM components controlling cellular adhesion. Integrins act as anchors by linking the matrix with the intracellular cytoskeleton, while availability of ECM binding sites, i.e. ECM composition and density, has been shown to regulate integrin clustering [33]. In comparison to mock controls, protein level of $\beta 3$ integrin was increased by $32 \%$ and of $\beta 1$ integrin by $127 \%$ in $\triangle$ pBK-ITIH5 clones (Fig. 5a and b) thus hypothesizing that ITIH5 expression modulates activation of downstream effectors of the integrin signaling cascade crosstalk like small G-proteins of the Rho subfamily. Activation of the antagonists Rac1 and RhoA was analyzed which has been reported to regulate different steps during cell movement and is thought being modulated by $\beta 1$ and $\beta 3$ integrin, respectively [34]. Interestingly, we found that the balance between the activity of RhoA and Rac1 shifted towards increased activation of RhoA in ITIH5 clones while Rac1 was abundantly activated in control cells (Fig. 5c and d).

Consequently a closer look on mesenchymal migration was taken by performing a wound healing assay. Forced ITIH5 expression inhibited cell migration of basal-type MDA-MB-231 cells, i.e. MDA-MB-231 mock clones repopulated the wounded area notably faster than corresponding ITIH5-expressing single-cell clones over 4 days. Impairment of MDA-MB-231 cell migration was confirmed by all analyzed MDA-MB-231 ITIH5 single-cell clones $(n=5)$ compared to the MDA-MB-231 WT and mock clones $(n=3)$. The mean cell motility rate of independent clones of both groups is shown in Fig. 5e. ITIH5-expressing clones were not able to detach from the peripheral edge of the confluent cell layer and to migrate as single-cells into the wound as shown for mock clones (Fig. 5f). Already 1 day after scratching, most mock clones had repopulated almost the entire wound (overall 86.3\%), whereas the MDA-MB-231 ITIH5 clones covered on average $43.6 \%$ of the wounded area (Fig. $5 \mathrm{~g}$ ). Interestingly, ITIH5 expression did not alter the migration of T47D $\triangle \mathrm{pBK}$-ITIH5 single-cell clones (data not shown) whose parental cell line is known to feature already a well-differentiated, epithelial-like phenotype.

Given that, the architecture of the actin cytoskeleton and focal adhesions was determined reflecting integrin clusters on the cell surface of MDA-MB-231 ITIH5 cells. $24 \mathrm{~h}$ after seeding ITIH5-expressing MDA-MB-231 cells formed cell clusters whose focal adhesions were found being close to the cell periphery and were less elongated. In contrast mock single-cells exhibited F-actin stress fibers passing through the cell body that are connected with elongated focal adhesion sites in the cell body (Fig. 5h). MDA-MB-231 ITIH5 clones showed less stress fiber formation but formed mostly cortical actin bundles, i.e. the F-actin is condensed around the cell periphery. As expected for those tightly organized cell clusters, single-cell polarization was impaired, i.e. cell polarization into a distinct protrusive front and a retracting rear as obvious in mock cells (illustrated in Fig. 5i). Instead, $\triangle \mathrm{pBK}$-ITIH5 cells remained in a tight cell cluster potentially connected by cell-cell contacts as upregulation of desmosomal cadherins was demonstrated. Real-time PCR analysis significantly confirmed increased expression of desmoglein-2 (DSG2, array-effect: FC: 2.04 ) by 7.2-fold, of desmocollin-2 (DSC2, array-effect: FC: 1.54) by 184.0 -fold and of desmoplakin (DSP, array-effect: FC: 1.91) by 24.8 -fold (Fig. 5j).

\section{ITIH5-driven phenotype switch of basal-type breast} cancer cells is associated with epigenetic reprogramming Facing the identified phenotype switch of aggressive breast cancer cells driven by ITIH5 expression, we focused on potential mechanisms. It has been suggested that cellular differentiation might impact on epigenetic regulation of gene expression [35] - particularly on DNA methylation [36-38]. Therefore, DNA methylation profiles of the MDA-MB-231 WT, mock (\#1 and \#2), and ITIH5 single-cell clones (\#4, \#7, and \#12) was analyzed using the Infinium HumanMethylation450 (450 K) BeadChip technology. We then selected those CpG sites with significant $(p<0.05)$ methylation differences (mean $\beta$ value) of more than $20 \%$ between mock (and WT) and ITIH5-expressing MDA-MB-231 clones: overall 1511 CpG sites passed this threshold (Fig. 6a) corresponding to 728 different genes. 695 of these genes are associated with a GO term including in particular those genes involved in cellular adhesion (e.g. GO:0098742, $p=2.98 \times$ $10^{-12}$ ) (Fig. 6b). Interestingly, these GO terms were nearly conformable with the GO annotations based on mRNA expression profiling (see Table 1). In addition highly significant enrichment of genes encoding for cellular components of the ECM (e.g. GO: 004442, $p=4.68 \times 10^{-4}$ ) was found including collagens such as COL2A1 or COL15A1. Using gene set enrichment analysis (GSEA) the most significant overlap was observed of hypo- and hypermethylated CpG sites located in a promoter region (TSS1500, TSS200, 5'UTR) (overall $n=404$ ) with genes, for instance, 1) containing around the TSS the motif CAGGTG which matches annotation for TCF3 $\left(p=2.26^{-20}\right)$, or 2) targeted by the Polycomb (PcG) protein SUZ12 $\left(p=1.66^{-15}\right)$ (Additional file 2).

Subsequently, hyper- and hypomethylated CpGs identified in ITIH5-expressing MDA-MB-231 clones were compared with a list of genes of embryonic stem cells (ES) that have H3K4Me3 and/or H3K27Me3 enrichment in a $5000 \mathrm{bp}$ region upstream and downstream from the transcription start site (TSS) based on previously published ChIP-seq data [39]. The methylation status was 

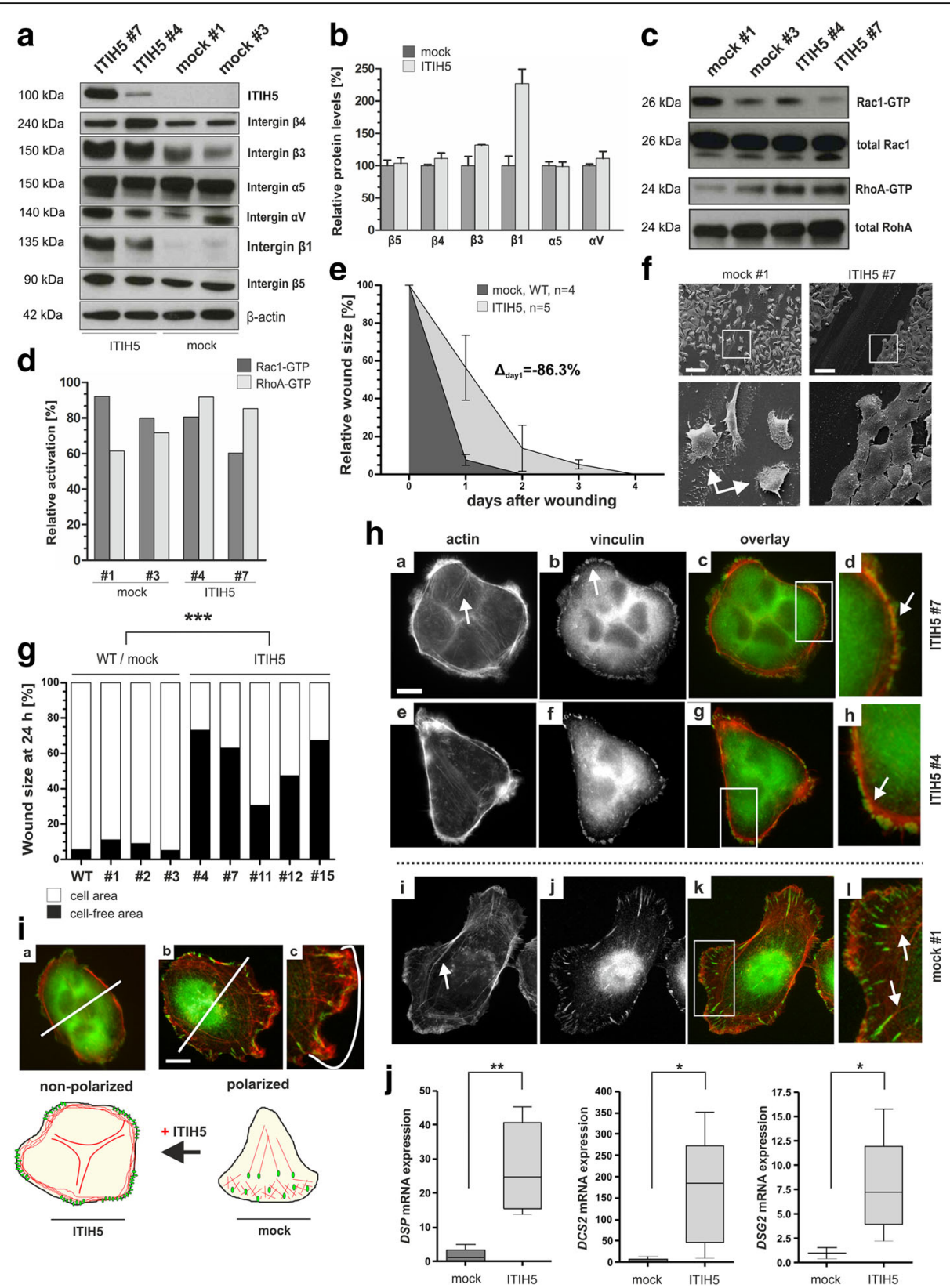

Fig. $5 \mathrm{ITH}$ H alters integrin signaling impairing single-cell polarization. a Integrin protein expression/stability in TTIH5 and mock clones. $\beta$-actin served as loading control. b Densitometric evaluation of western blot results demonstrating an integrin protein shift. Relative protein expression levels are normalized to $\beta$-actin. Mean protein level of mock clones was set to 100\%, respectively. c Analysis of integrin downstream signaling. Representative western blot results illustrate activated Rac1 and RhoA GTPases in two independent ITIH5 and mock clones. Total Rac1 and RhoA served as loading control. d Densitometric evaluation of GTPases activation. Relative protein expression levels are normalized to total Rac1 and total RhoA, respectively. Mean protein level of mock clones was set to 100\%. e Cell migration was analyzed by using a wound healing assay. Mean migration rate of a control cell set ( $n=4$, WT and mock clones) and ITIH5 MDA-MB-231 clones $(n=4)$ was analyzed over 4 days. Vertical lines: standard deviation (S.D.) of triplicates. Cell-free area on day 0 was set as $100 \%$ and used for standardization. $\Delta_{\text {day } 1}$ : differences of cell-free areas on day 1. $\mathbf{f}$ Documentation of the wounded area by SEM $24 \mathrm{~h}$ after scratching. Left rectangle regions: separately enlarged. Scale bar $=100 \mu \mathrm{m}$. g Detailed comparison of wound closure after $24 \mathrm{~h}$ for each single-cell clone. $\mathbf{h}$ Visualization of F-actin architecture and focal adhesion are shown of ITIH5 and mock clones. Upper rows: Representative micrographs of ITIH5 clone \#7 and ITIH5 clone \#4. White arrows indicate cortical actin bundles (red) and less elongated focal adhesions (green dots). Lower row: Representative micrographs of mock clone \#1. White arrows: F-actin stress fibers (red) co-localized with elongated focal-adhesion sites (green) in the cell body of single-cells. Scale bar $=10 \mu \mathrm{m}$. $\mathbf{i}$ lllustration of the ITIH5-associated impact on cell-polarization necessary for cell migration. a: ITIH5 clones showed tight clusters lacking cell polarization. b: mock cells are able to form a distinct protrusive front and a retracting rear. Scale bar $=10 \mu \mathrm{m} \mathbf{j}$ Real-time PCR analysis demonstrating significant upregulation of DSP, DSC2 and DSG2 in ITIH5 $(n=5)$ compared to mock clones $(n=4)$. Horizontal lines: grouped medians. Boxes: $25-75 \%$ quartiles. Vertical lines: range, peak and minimum; ${ }^{*} p<0.05,{ }^{* *} p<0.01,{ }^{* * *} p<0.001$ 

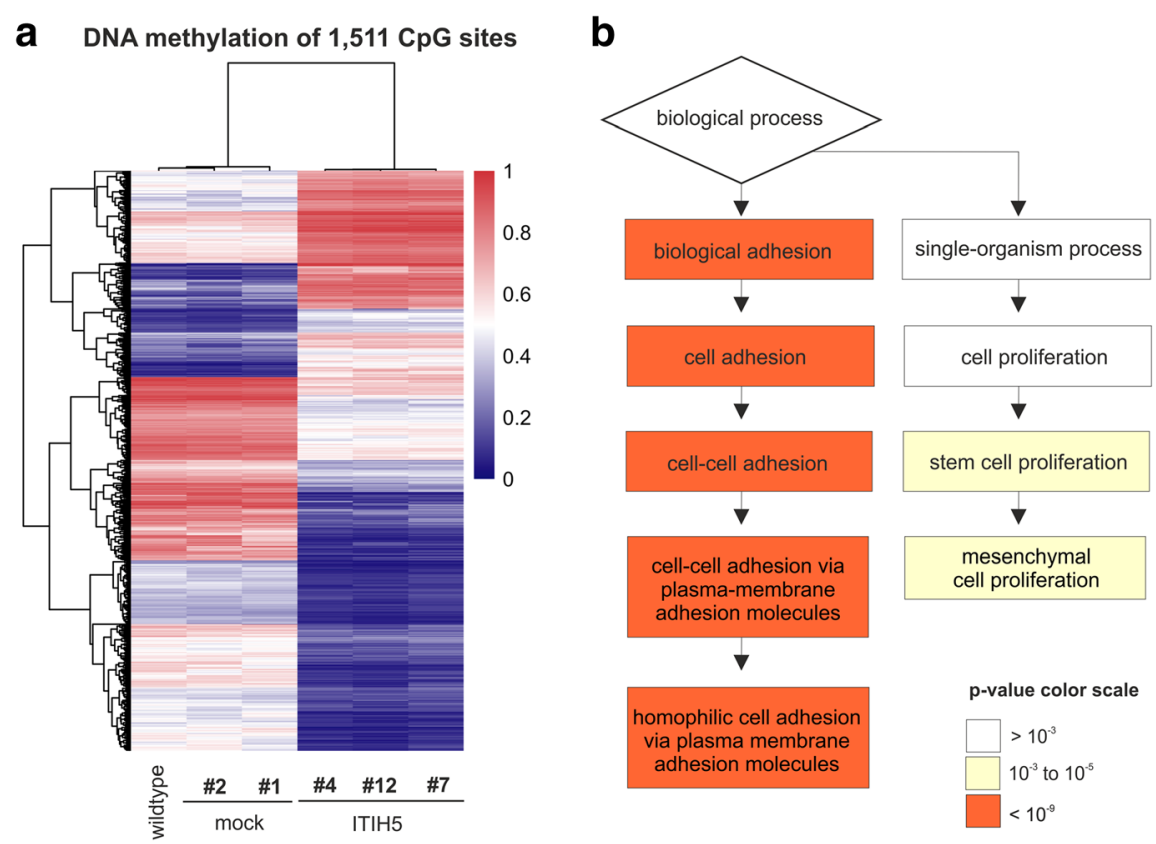

C DNA methylation of 242 promoter regions

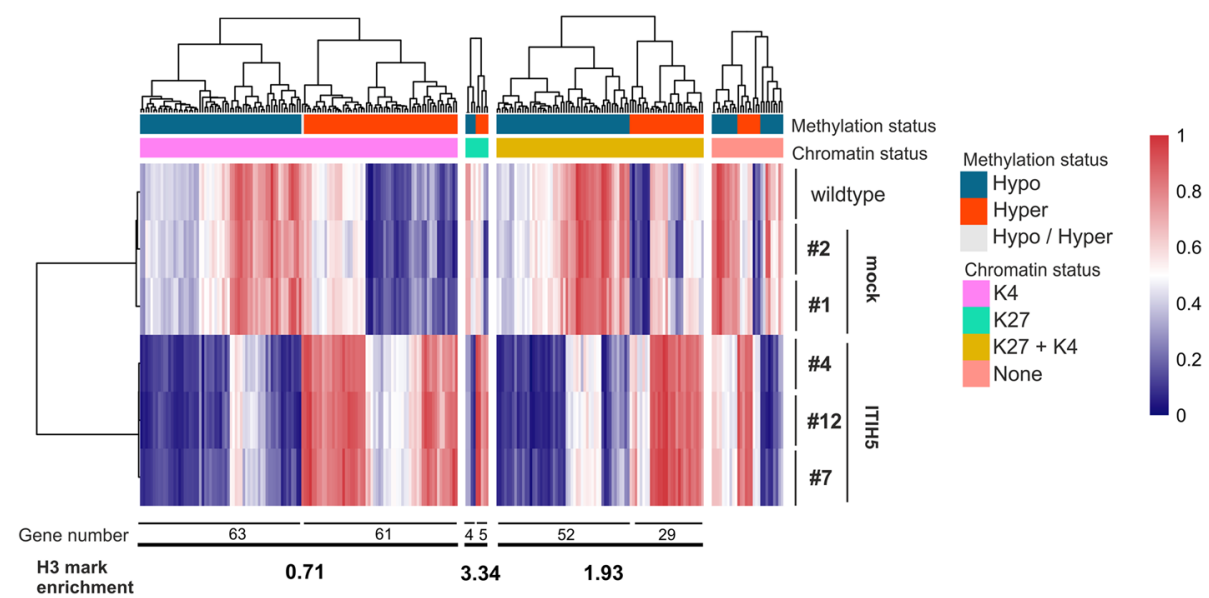

Fig. 6 Epigenomic reprogramming of genes involved in cell adhesion and proliferation triggered by ITIH5 expression. Analysis of the DNA methylation profiles of the MDA-MB-231 WT, mock (\#1 and \#2), and ITIH5 transfected MDA-MB-231 ITIH5 single-cell clones $(4,7$, and \#12) using the Infinium Human Methylation450 (450 K) BeadChip technology. a Heatmap of 1512 CpG sites exhibiting significant $(p<0.05, \Delta \beta$-value $>0.2)$ methylation alterations between MDA-MB-231 WT, mock and ITIH5 single-cell clones demonstrated an epigenetic reprogramming of MDA-MB-231 cancer cells. b Cartoon illustrating gene ontology analysis of annotated 695 genes corresponding to the 1511 CpG sites. c Heatmap analysis of 242 hyper- or hypomethylated $\mathrm{CpG}$ sites (out of identified $1511 \mathrm{CpGs}$ ) located in potential regulatory promoter regions that are associated with H3K4Me3 and/or H3K27Me3 enrichment in a 5000 bp region upstream and downstream from the TSS. Chromatin status: $\mathrm{K} 27=\mathrm{H} 3 \mathrm{~K} 27 \mathrm{Me} 3, \mathrm{~K} 4=\mathrm{H} 3 \mathrm{~K} 4 \mathrm{Me} 3$

determined in 14,356 promoter regions characterized by $\mathrm{Ku}$ and colleagues comprising a minimum of $5 \mathrm{CpG}$ sites (Additional file 3). 274 CpG sites out of 1511 were classified according to 242 different corresponding promoter regions (Fig. 6c, Additional file 4). 214 promoters featured a significant $\left(p<10^{-6}\right)$ association with a potential H3 methylation status described for ES cells. Interestingly, regions associated with the potential PcG signature H3K27Me3 were significantly enriched by 3.3fold (Table 2) in MDA-MB-231 ITIH5 clones. Promoters with a combined, i.e. with a potentially bivalent, H3K4Me3 and H3K27Me3 status were also enriched by 1.9-fold whereas regions associated with a potential H3K4Me3 status were under-represented in this data set. Hence, the identified epigenetic shift caused by ITIH5 involves promoters potentially associated with 
Table 2 Enrichment of differently methylated promoter regions potentially harboring histone $\mathrm{H} 3$ modifications described by $\mathrm{Ku}$ et al. [39]

\begin{tabular}{llll}
\hline & $\begin{array}{l}\text { Number of } \\
\text { regions }\end{array}$ & $\begin{array}{l}\text { Differential } \\
\text { methylated }^{*}\end{array}$ & $\begin{array}{l}\text { Relative } \\
\text { enrichment }\end{array}$ \\
\hline K4 & 9118 & $124(1.36 \%)$ & 0.71 \\
K27 & 140 & $9(6.43 \%)$ & 3.34 \\
K4+K27 & 2176 & $81(3.72 \%)$ & 1.93 \\
None & 1130 & $28(2.48 \%)$ & 1.29 \\
Total & 12,564 & $242(1.93 \%)$ & \\
\hline * $P<10^{-6}$ according to Fiser's exact test & &
\end{tabular}

bivalent chromatin that may be causative for a dynamic restoration and/or silencing of gene expression.

\section{DNA demethylation of distinct promoter regions is} associated with re-expression of the tumor suppressor gene DAPK1

We hypothesized that the identified shift in DNA methylation pattern influences expression of genes contributing to the ITIH5 induced, tumor suppressive phenotype of MDA-MB-231 cells. Therefore, we had a closer look on the gene expression pattern associated with ITIH5. By applying a class comparison analysis between control cell populations (mock clones) and ITIH5-transfected clones, we aimed at identifying the strongest co- and anti-regulated genes which met the following criteria: Significantly $(p<0.05)$ differentially expressed with a minimal change in expression by 3 -fold. Significantly up- and downregulated genes are summarized in Table 3. While tumor promoting genes such as AGR2 were downregulated, known tumor suppressor genes like NDRG2 and DAPK1 were upregulated 4.3- and 4.6-fold, respectively.

DAPK1 expression was furthermore verified on mRNA as well as on protein level in $\triangle \mathrm{pBK}$-ITIH5 breast cancer cells (Fig. 7a). By comparing the profiled DNA methylation of significant $\mathrm{CpG}$ sites and the expression signature, a clear demethylation of CpG sites within the 5' UTR region close to the transcription start site (TSS) of the DAPK1 gene in $\triangle \mathrm{pBK}-\mathrm{ITIH} 5$ clones (Fig. $7 \mathrm{~b}$ ) was shown. Within this upstream promoter region (ENSEMBL contig ENSG00000196730) a CpG-rich island between genomic positions $90,112,413$ and $90,114,138$ (+270 bp to +1725 bp relative to the expected TSS) on chromosome $9 q$ was verified which met the following criteria according to $\mathrm{Li}$ et al. [40]: DNA region: $\geq 200 \mathrm{bp}$; Obs/Exp: $\geq 0.6$; $\% G C: \geq 50$. This promoter region corresponds to the 242 identified promoters showing a significantly altered methylation status and is potentially marked by an activating H3K4Me3 histone modification (see Additional file 4). Performing Genomatix data base analysis [41] putative transcription binding sites in this 5'UTR locus were
Table 3 Genes 3-fold up-/downregulated by ITIH5

\begin{tabular}{lccl}
\hline Symbol & Fold-change & Parametric $p$-value & Regulation \\
\hline TNS4 & 0.13 & 0.0121 & down \\
CHRDL1 & 0.21 & 0.0306 & down \\
AREG & 0.22 & 0.0166 & down \\
AGR2 & 0.23 & 0.0169 & down \\
GLB1L2 & 0.26 & 0.0455 & down \\
TMEM163 & 0.29 & 0.0237 & down \\
ZG16B & 0.32 & 0.0139 & down \\
TPTE & 3.45 & 0.0114 & up \\
NDRG2 & 4.29 & 0.0002 & up \\
GJA5 & 4.34 & 0.0384 & up \\
GALNT14 & 4.44 & 0.0011 & up \\
DAPK1 & 4.55 & 0.0485 & up \\
COX7B2 & 5.05 & 0.0094 & up \\
PLCB4 & 5.55 & 0.0047 & up \\
GMFG & 6.51 & 0.0377 & up \\
THY1 & 8.31 & 0.0089 & up \\
ENG & 9.00 & 0.0019 & up \\
LCP1 & 14.83 & 0.0121 & up \\
\hline
\end{tabular}

determined with highly statistical reliability, namely SP1F (matrix similarity: 0.941), SMAD (matrix similarity: 0.963) and TF2B (matrix similarity: 1.0). In contrast to the 5'UTR region, CpG sites located within the DAPK1 gene body were clearly hypermethylated when compared to mock control clones (Fig. 7b).

By performing both methylation-specific PCR (MSP) (Fig. 7c) and pyrosequencing (Fig. 7d to e) decreased methylation level within the $\mathrm{CpG}$ island closely associated to the TSS of DAPK1 was subsequently confirmed. Based on pyrosequencing the methylation status of 14 individual CpG sites was analyzed demonstrating completely hypomethylated CpG sites within the 5'UTR region of DPAK1 in $\triangle \mathrm{pBK}-\mathrm{ITIH} 5$ cells. The median $D A P K 1$ methylation level of $\triangle \mathrm{pBK}$-mock clones $(n=3)$ was $26 \%$ featuring a high range between 12.5 and $50.5 \%$, whereas the median methylation of ITIH5 clones $(n=4)$ was consistently decreased in all analyzed ITIH5expressing clones $(n=4)$ down to $1 \%$ (mean: $1.3 \%$, s.d. \pm 1.4\%; range $0.0-1.5 \%)$. Overall DAPK1 methylation was decreased in median by $96.8 \%$ (Fig. 7e).

Next, decreased DAPK1 promoter methylation was demonstrated $72 \mathrm{~h}$ after application of demethylation drugs to mock control cells (clone \#2). The median methylation level of the analyzed DAPK1 5'UTR region was reduced from 67 to $53 \%$ (Fig. 8 a to b) in mock controls over 3 days. A representative diagram illustrating the methylation level of all 14 analyzed CpGs in mock cells, before and after DAC and TSA 


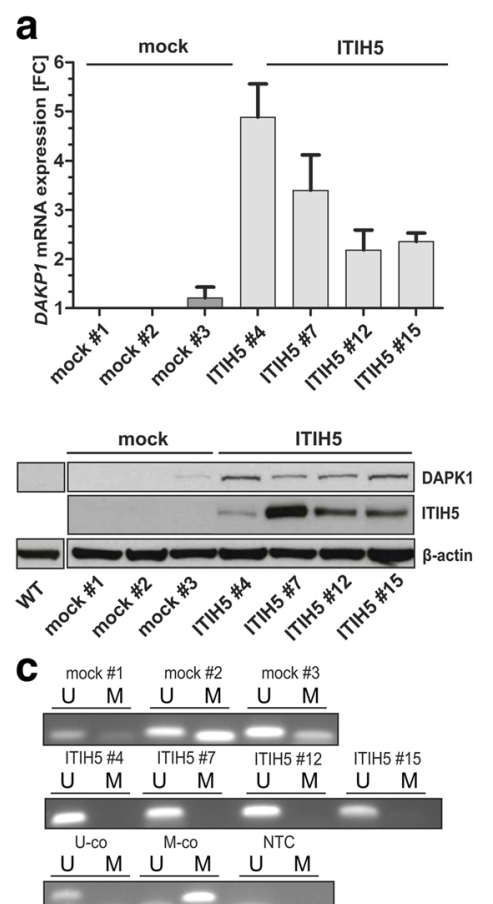

d

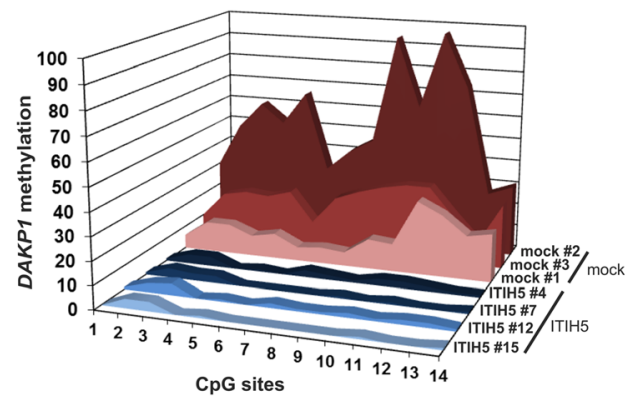

b 90,113,814 $\quad$ chr9 (q21.33) $90,278,261$
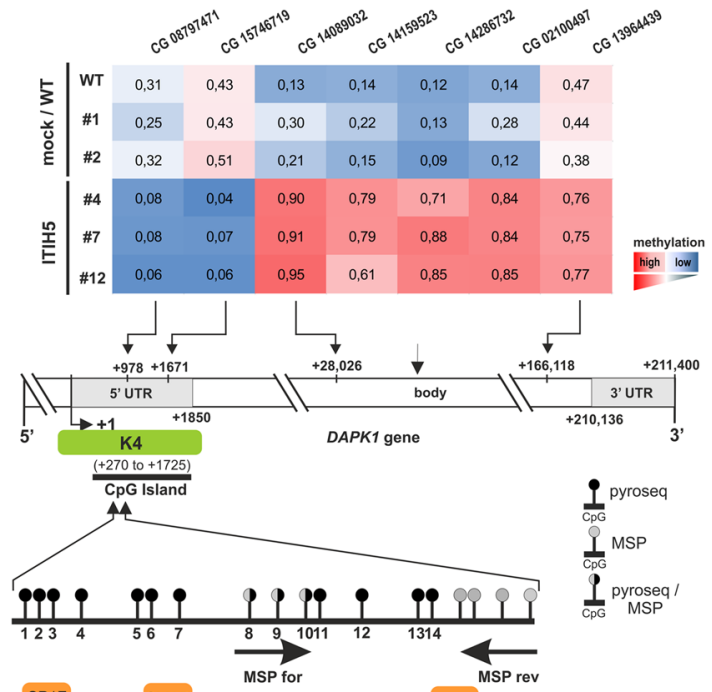
SP1F SMAD

e

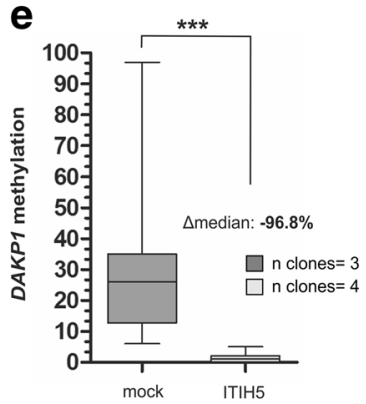

Fig. 7 ITIH5 mediates demethylation of the DAPK1 promoter 5'UTR region leading to its re-expression in basal-type breast cancer cells. a DAPK1 re-expression was confirmed in ITIH5 clones $(n=4)$ by real-time PCR (upper graph) and western blot analysis (lower images) when compared with and mock clones $(n=3)$ and MDA-MB-231 WT. $\beta$-actin served as loading control. $\mathbf{b}$ Schematic map of the human DAPK1 gene including the relative positions and $\beta$-values of $\mathrm{CpG}$ dinucleotides measured by $450 \mathrm{~K}$ methylation array profiling in MDA-MB-231 WT cells, mock and ITIH5 single-cell clones. Red: high methylation, blue: low methylation. +1: DAPK1 transcription start site (TSS). A predicted CpG island is located between genomic positions $90,112,413$ and $90,114,138$ (+270 bp to +1725 bp relative to the expected TSS) within the $5^{\prime}-U T R$ region. At this site a potential activating H3K4Me3 (K4) histone modification as mapped by Ku et al. [41] was described. The relative positions of $18 \mathrm{CpG}$ sites analyzed either by MSP (used primer: black arrows) and / or pyrosequencing within the DAPK1 5'UTR region are indicated. Three putative transcription binding sites in this gene locus were statistically identified: SP1F (matrix similarity: 0.941), SMAD (matrix similarity: 0.963) and TF2B (matrix similarity: 1.0). c DNA methylation of the DAPK1 5'UTR locus verified in mock and ITIH5 single-cell clones by using MSP. Band labels with $U$ and M represent an unmethylated and methylated DNA region, respectively. Bisulfite-converted unmethylated, (U-co) and polymethylated, genomic (M-co) DNA were used as controls. NTC: non-template control. d-e Quantification of DAPK1 5'UTR DNA methylation frequency by using pyrosequencing. d 3D graph illustrates methylation level for each analyzed CpG site (overall $14 \mathrm{CpGs}$ ) within the DAPK1 $5^{\prime}$ UTR locus in mock $(n=3)$ and ITIH5 $(n=4)$ single-cell clones. e Box plot analysis demonstrates significant reduction of the median methylation ratio within the DAPK1 $5^{\prime} U T R$ region in $\triangle \mathrm{pBK}-\mathrm{TTIH} 5$ compared to $\triangle \mathrm{pBK}$-mock clones. Horizontal lines: grouped medians. Boxes: $25-75 \%$ quartiles. Vertical lines: range, peak and minimum; ${ }^{*} p<0.05,{ }^{* *} p<0.01,{ }^{* * *} p<0.001$

treatment is shown in Fig. 8a. As a consequence of the reduced methylation level, upregulation of DAPK1 mRNA expression was observed in mock tumor cells after demethylation treatment (Fig. 8c) whereas no further DAPK1 expression was shown in ITIH5 clone \#4 harboring already an unmethylated
$D A P K 1$ promoter region as shown in Fig. $7 \mathrm{~d}$. In mock control cells, only treatment of both DAC and TSA leads to a maximum of DAPK1 mRNA reexpression by more than 1500 -fold. These findings support our notion that epigenetic alterations of the $D A P K 1$ promoter may be caused by synergistic 


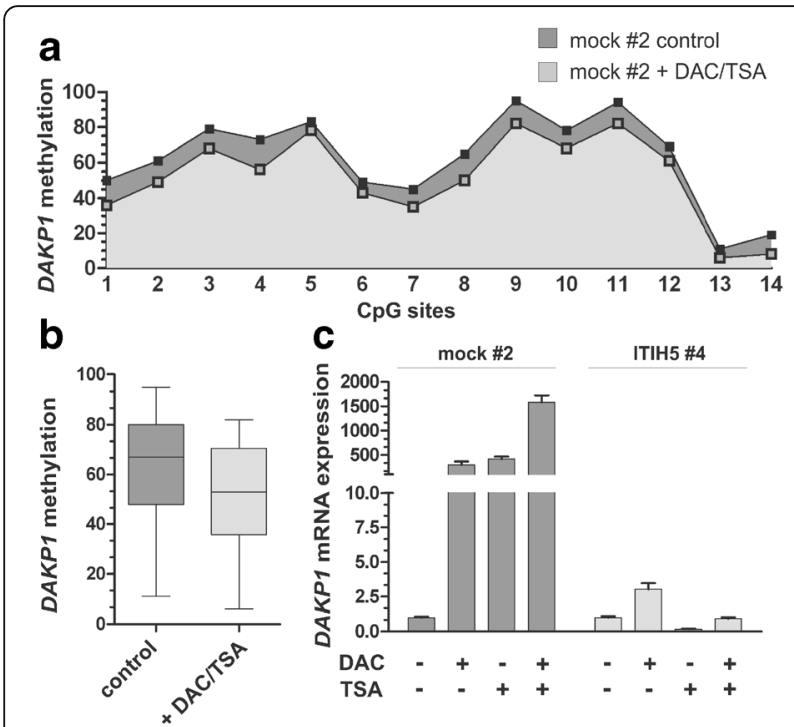

Fig. 8 In vitro demethylation of the DAPK1 5'UTR locus correlates with DAPK1 re-expression in $\triangle \mathrm{pBK}$-mock cells. a Pyrosequencing analysis for each CpG dinucleotide (1-14) within the DAPK1 5'UTR region determined prior (-DAC/-TSA; dark-grey-filled) and after in vitro demethylation treatment (+DAC/+TSA; grey-filled). b Box plot analysis shows reduction of the median methylation ratio within the DAPK1 5'UTR region in $\triangle \mathrm{pBK}$-mock cells after DAC/TSA treatment (+) compared to non-treated cells (control). Horizontal lines: grouped medians. Boxes: $25-75 \%$ quartiles. Vertical lines: range, peak and minimum; ** $p<0.01$. c Real-time PCR results illustrate a clear DAPK1 re-expression after treatment with both DAC and TSA (+) in mock clones while now further expression of DAPK1 mRNA was detected in ITIH5 clones already harboring an unmethylated DAPK1 5'UTR region. Non-treated cells (-DAC,-TSA) were set to 1, respectively. Error bars: + s.e.m

crosstalk between DNA methylation and histone modification that has a major impact on the regulation of DAPK1 re-expression.

\section{Knockdown of DAPK1 promotes tumor cell migration in MDA-MB-231 $\triangle \mathrm{pBK}-\mathrm{ITIH} 5$ cells}

As DAPK1 (death-associated protein kinase (DAP Kinase)) is a well-known tumor suppressor [42], we aimed to demonstrate whether its re-expression may explain some of the ITIH5-associated suppressive attributes in basal-type breast cancer cells. DAPK1 has been shown to mediate apoptosis but accumulating studies showed involvement of DAPK1 in integrin signaling impairing cell migration [43]. RNA interference-mediated DAPK1 knockdown was performed in ITIH5-expressing MDAMB-231 cells (clone \#7) applying two different siRNA sequences (\#1 and \#2) alone as well as in combination (Fig. 9a and b). Interestingly, based on a caspase 3 activity assay, a clear apoptotic resistance of transfected cells was not observed after DAPK1 knockdown (data not shown) that is consistent with a previous report in this cell line [44]. In turn, using a monolayer wound healing assay
siRNA-mediated knockdown of DAPK1 clearly increased tumor cell migration of $\triangle \mathrm{pBK}$-ITIH5 clone \#7 compared to corresponding cells transfected with the non-silencing control siRNA (nc-control siRNA) that served as negative control (Fig. 9c and d). Stably ITIH5-expressing MDAMB-231 cells transfected with both DAPK1 siRNA sequences nearly repopulated the entire wounded cell-free area (siRNA \#1: 100\%, siRNA\#2: $89.71 \%$, siRNA \#1+ \#2: $96.35 \%)$ after 48 h, i.e. $\triangle$ pBK-ITIH5 cells with reduced DAPK1 expression tend to restore motile characteristics as observed for MDA-MB-231 WT cells (see Fig. 5g). In contrast to that, nc-control siRNA transfected ITIH5expressing clones had repopulated only $67.01 \%$ of the wound area at this time point. These data clearly demonstrate that the ITIH5-DAPK1 molecular axis plays an important role in the regulation of MDA-MB-231 cell motility.

\section{Discussion}

Previously, we revealed that loss of ITIH5 expression caused by aberrant promoter hypermethylation is associated with poor prognosis and clinical correlates of metastasis in breast cancer [16, 23]. In the current study, ITIH5 downregulation was abundantly found in distant metastases and intrinsic subtypes associated with poor prognosis, i.e. luminal B, HER2-enriched and basal-like breast cancer. ITIH5 loss predicted shorter overall survival of patients with non-metastatic tumors proposing a prominent role of ITIH5 especially in tumors which tend to metastasize early and whose disease management and personalized therapy is still insufficient. To give insight into ITIH5 biology going beyond the assumed role as a prognostic biomarker in breast carcinomas, we established two different stable gain-of-function models, i.e. weak-aggressive T47D and metastatic MDA-MB-231 single-cell clones overexpressing full-length ITIH5. In both cell lines ITIH5 mediated suppression of colony and cell growth while only in luminal-type T47D cells ITIH5-triggered increased programmed cell death. However, this is consistent with our recent finding in luminal-like RT112 bladder cancer cells due to ITIH5 re-expression [18]. These data indicate that ITIH5 may control mechanisms to reduce cancer cell growth independently of a given tumor subtype or entity similar to the described function of ITIH1-3 by stabilizing ECM integrity $[9,45,46]$.

In MDA-MB-231 breast cancer cells ITIH5 induced a phenotypic switch, which to our knowledge has not yet been reported for any member of the ITI protein family before. Originally metastatic cancer cells underwent an epigenetic shift driven by ITIH5 that cause a distinct signature of expressed genes. Among others, re-expression of known tumor suppressor genes such as DAPK1 [42] was clearly demonstrated. As a consequence, forced 


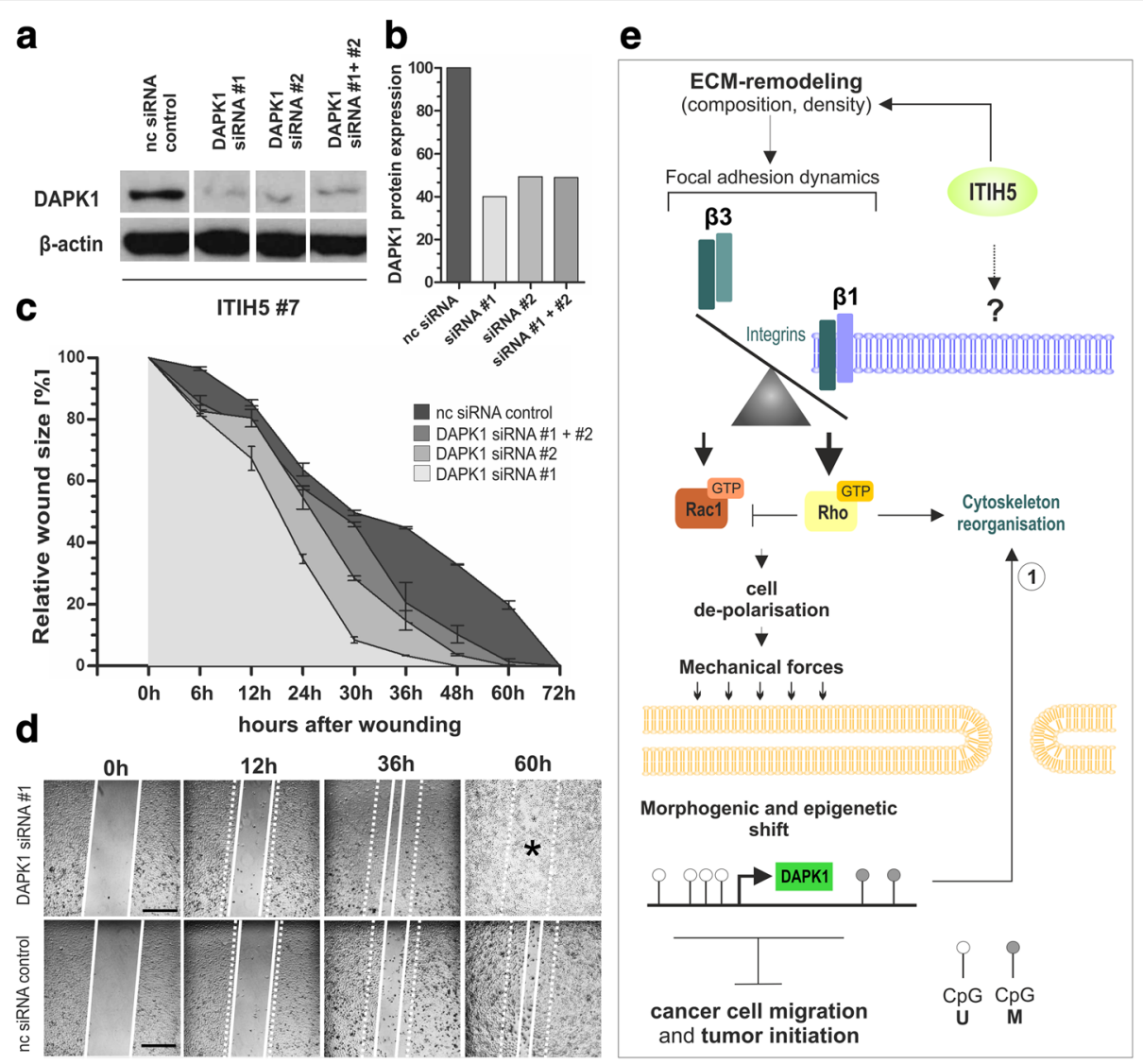

Fig. 9 DAPK1 knockdown restore a motile phenotype in $\triangle \mathrm{pBK}$-ITIH5 cells in vitro. a DAPK1 protein expression in ITIH5-expressing cells $48 \mathrm{~h}$ after transfection with DAPK1-siRNA \#1, DAPK1-siRNA \#2 as well as \#1 and \#2 combined in comparison to nc siRNA transfected control cells. $\beta$-actin served as loading control. b Densitometric determination of DAPK1 protein knockdown in $\triangle \mathrm{pBK}-\mathrm{ITIH} 5$ cells compared to control. c Cell migration of ITIH5 clones after treatment with DAPK1-siRNAs was analyzed by using a wound healing assay over $72 \mathrm{~h}$. nc siRNA transfected cells served as negative control. Vertical lines: standard deviation (S.D.). Cell-free area on day 0 was set as $100 \%$ and used for standardization. d Representative wound area documentation by light microscopy of DAPK1-siRNA \#1 and nc siRNA control 0, 12, 36, and $60 \mathrm{~h}$ after scratching. White line: cell-free wound area. White dashed line: original wound area size at $0 \mathrm{~h}$. Scale bar: $500 \mu \mathrm{m}$. e Working model highlighting factors potentially involved in ITIH5-driven phenotype shift of mesenchymal MDA-MB-231 breast cancer cells towards an epithelial-like state. ITIH5 remodels the ECM that is accompanied by changes in integrin composition. As a consequence downstream signaling is shifted towards RhoA activation. Clustered cancer cells further lacked polarization but featured in turn strong cell-matrix adhesion and modulated biomechanical cues. Re-expression of DAPK1, caused by epigenetic reprogramming, may be finally involved in ITIH5 mediated suppression of tumor cell migration dynamics potentially by re-organization of cytoskeleton structures as recently described (1): [43, 44]

ITIH5 expression led to a remarkable low-aggressive phenotype causing a reduction of lung colonies in vivo. As metastases were almost exclusively found in lungs of mice injected with cancer cells lacking ITIH5 expression, impaired tumor initiation capabilities could be suggested, a feature mainly attributed to CSC.

Mechanistically, ITIH5 expression was associated with regulation of genes involved in categories of cell adhesion and cell differentiation. Matrix adhesion of $\Delta \mathrm{pBK}-$ ITIH5 cells was significantly enhanced on physiologically coated substrates, mimicking the basement membrane (BM). ITIH5 also altered the composition of such specialized ECM structures as the BM constituent collagen type IV was identified being upregulated. According to this, profound changes in expression of integrin cell surface receptors were demonstrated that are known to bind to the $\mathrm{BM}$ being involved in controlling cell adhesion and migration [34, 47]. Because of their outside-in-signaling capacity, integrins function not only as regulators of cell adhesion but also as sensors of their extracellular environment regulating downstream signaling [48] and it is likely that they have completely different effects on behavior of cancer cells, depending on which integrin receptors and ligands are exposed [49]. Alterations in the profile of integrin expression as identified in ITIH5 clones have been reported to cause dramatic shifts in modes of cell migration [34]. In particular the balance between $\beta 1$, a putative metastasis suppressor in human cancer [50], and $\beta 3$ integrin is thought to play a critical role [51]. 
Interestingly, increased $\beta 3$ integrin was observed due to ITIH5 re-expression in MDA-MB-231 cells. Nevertheless, $\beta 1$ integrin, which is almost not expressed in mock clones, is even stronger induced in ITIH5 clones so that the balance between $\beta 3$ and $\beta 1$ integrin was clearly shifted towards $\beta 1$. While $\beta 3$ integrin has been reported being associated with Rac1 activation, $\beta 1$ integrin regulates in particular RhoA activity [34]. This notion is important because Rac1 facilitates F-actin polymerization and locally decreases cell-membrane tension that lead to lamellipodia formation during the first step of cell migration. Its activity is blocked by RhoA GTPases in the second phase of cell migration regulating actomyosin contractility [52].

Already in 2005, Danen et al. reported that integrin $\alpha \mathrm{V} \beta 3$ promotes directional cell migration in the absence of integrin $\alpha 5 \beta 1$ being characterized by a single large lamellipodium and lower RhoA activity [53, 54] as also obvious in mock control cells. In turn, $\alpha 5 \beta 1$ is particularly efficient at promoting later phases of cell spreading by supporting strong RhoA-mediated contractility and random migration. In our $\triangle \mathrm{pBK}$-ITIH5 model we showed that ITIH5-expressing MDA-MB-231 cells were not able to disseminate from neighboring cells moving as single-cells directional into the wounded area. As a consequence ITIH5-expressing significantly higher contractile cell forces compared to their mock clones. This result is in good agreement with the simultaneous upregulation of active RhoA-GTPases in ITIH5 clones, which are known to mediate matrix adhesiondependent cell forces via Rho/Rock signaling cascades [55] giving a mechanistic explanation for the highadhesive, well-differentiated phenotype. These findings were associated with clustering of $\Delta \mathrm{pBK}$-ITIH5 cells and with reduced polarization into a distinct protrusive front and a retracting rear end. Truong et al. have recently reported that functional inhibition of $\beta 1$ integrin converted the migratory behavior of human triple-negative breast cancer (TNBC) cells from collective to single-cell movement facilitating lung colonization in vivo [56]. Moreover, $\beta 1$ integrin promotes an epithelial phenotype in those TNBC cells by restoring, for instance, E-cadherin expression in a TGF- $\beta$ dependent manner. Hence, upregulation of desmosomal components like DSP and DSC2 linking neighboring cells may contribute to tightly organized colony structures of ITIH5-expressing MDA-MB-231 cells impairing mesenchymal single-cell migration.

It is astonishing that expression of a single ECM factor in vitro, i.e. ITIH5, can effect hyper- or hypomethylation of more than $1500 \mathrm{CpG}$ sites in metastatic cancer cells. The term "epigenetic reprogramming" is commonly used to describe profound alterations in the epigenetic makeup (e.g. [57, 58]) - and therefore appears to be justified in this context. Addressing the question why those DNA regions showed differences in DNA methylation, we focused on mechanisms known to be involved in regulating DNA methylation dynamics. So far increasing evidence suggest that histone modifications, namely H3K27Me3 and H3K4Me3, and associated PcG and trithorax-group (trxG) proteins are not only critical for changes in gene expression upon embryonal stem (ES) cell differentiation [59], but also for development of cancer (stem) cells [60-63]. Cross talk between histone methylation marks and DNA methylation is thought to regulate DNA methylation dynamics via recruiting proteins like DNA methyltransferases (DNMTs) [64]. In agreement with that, GSEA analysis revealed highly significant enrichment of genes harboring targets of the Polycomb protein SUZ12. By correlating corresponding CpG positions with histone modification marks as described by $\mathrm{Ku}$ et al. [39], 214 promoters were identified that have been previously reported being marked by either H3K4Me3 and/or H3K27Me3 in ES cells and have changed their DNA methylation status in ITIH5 clones. Importantly, genes associated with both H3K27Me3 alone and a combined, i.e. with a potentially bivalent H3K4Me3 and H3K27Me3 status, were significantly overrepresented. Thus, enrichment of promoter regions associated with dynamics in $\mathrm{H} 3$ methylation could indeed contribute to the epigenetic shift allowing distinct DNA demethylation patterns as observed for the DAPK1 5'UTR sequence close to the TSS.

DAPK1 is a calmodulin-regulated and cytoskeleton associated serine/threonine kinase $[65,66]$. Accumulating evidence suggest that DAPK1 plays an important role in tumor suppression. Epigenetic silencing of $D A P K 1$ has been demonstrated to correlate with higher risk for recurrence and metastasis in various tumor entities [42]. DAPK1 is a pro-apoptotic factor (e.g. [67]) that abrogates matrix survival signals by inside-out inactivation of $\beta 1$ integrin impairing the p53-apoptosis pathway [68]. Aside of its apoptotic function Kuo and colleagues postulated an apoptosisindependent mechanism of DAPK1, i.e. uncoupling of stress fibers and focal adhesions by modulation of integrin adhesion [43]. This study fits to our observation that the cytoskeleton was re-organized in DAPK1-expressing $\triangle \mathrm{pBK}-\mathrm{ITIH} 5$ cells. It has been shown that DAPK1 mediates a disruption of the cell polarity by blocking the Rho-GTPases cdc42 in MDAMB-231 cells leading to inhibition of cell migration in a wound healing assay [44]. Consistent with that, knockdown of DAPK1 had restored motile capacities, at least in part, of ITIH5-expressing MDA-MB-231 cells, indicating involvement of DAPK1 in the RhoAB1-integrin-mediated signaling axis. A cartoon summarizing these finding is illustrated in Fig. 9e. 
Underlying mechanisms of the epigenetic shift induced by ITIH5 in basal-type breast cancer cells and the putative role of specific ECM components and receptors appear complex, and must be addressed in future studies. As luminal T47D cells already grow in epithelial-like clusters, it makes sense that ITIH5 did not trigger a similar effect in those already well-differentiated tumor cells. Beyond that different settings of cell-surface receptors might explain a responsibility for ITIH5-mediated functions such as HA-crosslinking in dependence of a given background. For instance, MDA-MB-231 cells highly express CD44, a known HA-receptor facilitating metastatic CSC-like features [69], whereas T47D has been previously characterized as CD44 ${ }^{\text {low }}$ [70]. Since Mina Bissell postulated a profound impact of the ECM and regulatory proteins on cell differentiation [1] already in 1982 [71], it is by now well described that epigenetic gene expression control such as chromatin remodeling $[2,72]$ can be orchestrated by signals from the cellular microenvironment. Biomechanical cues as modified by ITIH5 are thought to contribute to global internal organization of nuclei $[73,74]$ controlling chromatin structure [36]. Irrespective of that our data underline the complex but fundamental effects of the ECM and its constituents on cell phenotypes and differentiation in the context of malignant progression.

\section{Conclusions}

In the current study, we provide evidence that the ECM modulator ITIH5 suppresses tumor cell migration and colonization of metastatic MDA-MB-231 breast cancer. As a result of an epigenetic reprogramming driven by ITIH5, tumor suppressor genes such as DAPK1 were reexpressed reversing the aggressive phenotype. Bearing in mind that MDA-MB-231 cells have been shown displaying CSC properties [75, 76], the shift of ITIH5expressing MDA-MB-231 cancer cells towards an epithelial-like differentiation state accompanied by an inability to initiate high number of metastases in vivo suggests impairment of metastatic characteristics.

\section{Methods}

\section{Animals}

Female BALB/c ${ }^{\text {nu/nu }}$ mice were purchased from Charles River Laboratories International (Wilmington, MA). All animal procedures and experiments were conducted in accordance with the German federal law regarding the protection of animals. The respective protocols were approved by the administration of the "Landesamt für Umwelt, Natur und Verbraucherschutz" (LANUV, Recklinghausen, Germany - AZ 87-51.04.2010.A226). For the care of laboratory animals, Guide for the Care and Use of Laboratory Animals (National Institutes of Health publication 86-23, 1985 revision) was followed.

\section{TCGA data set}

Data from breast cancer, normal and metastatic tissues were used from The Cancer Genome Atlas (TCGA) [25], comprising overall patients' data of an independent platform: Gene expression IlluminaHiSeq $(n=1215)$. The data of this study can be explored using the cBio Cancer Genomics Portal (http://cbioportal.org).

\section{Cell lines and reagents}

Breast cancer cell lines T47D and MDA-MB-231 were obtained from the American Type Culture Collection (ATCC, Manassas, VA), which assures molecular authentication of cell] lines [77], and was resuscitated before using in experiments. Otherwise cell lines were authenticated, within 12 months of being used in the study and were cultured as described previously [78] and regularly tested for mycoplasma infection using the PCR-based Venor GeM Mycoplasma Detection Kit (Minerva Biolabs, Berlin, Germany).

\section{Transfection and single-cell cloning of T47D and MDA-MB-231 cells}

Transfection of both T47D and MDA-MB-231 cells with ITIH5-pBK-CMV expression vector, containing the fulllength human ITIH5 cDNA derived from normal breast tissue, was performed as recently described [16]. Singlecell clones were selected by limited dilution under geneticin (G418) pressure (T47D: $400 \mu \mathrm{g} / \mathrm{ml}$; MDA-MB-231: $1000 \mu \mathrm{g} / \mathrm{ml})$.

\section{RNA interference of DAPK1}

Human $\triangle$ pBK-ITIH5 and mock clones were transfected with HiPerfect transfection reagent (Qiagen) applying two siRNA sequences directed against DAPK1 alone (\#1: Hs_DAPK1_6, Cat. No. SI02223781, 5'-CGGCTATTA CTCTGTGGCCAA -3' and \#2: Hs_DAPK1_6, Cat. No. SI02223774, 5'- AAGCATGTAATGTTAATGTTA.-3' (20 $\mathrm{nM}$ each)), or in combination of both according to the manufacturer's instructions. Cells were treated every $48 \mathrm{~h}$ with siRNA sequences to ensure sufficient DAPK1 knockdown. Commercial non-silencing control siRNA (nc siRNA) (5'-AATGCTGACTCAAAGCTCTG-3') served as negative control. Knockdown was verified by RT-PCR and western blot analysis after 48, 96 and 144 h. Functional studies were started immediately after $48 \mathrm{~h}$ siRNA treatment.

\section{Nucleic acid extraction and reverse transcription PCR}

Total cellular RNA from cultured cells and tumor nodules of mice lungs (samples pooled for test group) was prepared by using TRIzol reagent (Invitrogen). cDNA was synthesized using the reverse transcription system (Promega, Madison, WI) as previously described [16]. 


\section{Real-time PCR}

cDNAs were amplified by real-time PCR using SYBRGreen PCR mix (Bio-Rad Laboratories, Munich, Germany) performed in an iCycler IQ5 (Bio-Rad Laboratories) and quantified by the comparative $\mathrm{C}_{\mathrm{T}}$ method calculating relative expression values as previously described [79]. All used primers spanned at least one intron, and are listed in Additional file 5.

\section{In vitro demethylation}

Whole-genome demethylation of human stable MDAMB-231 clones was performed as recently published [80]. In brief, demethylation agent 5-aza-2'-deoxycytidine (DAC) was added to a final concentration of $5 \mu \mathrm{M}$ on days 1,2 and 3 . On day 3 cells were additionally treated with $300 \mathrm{nM}$ trichostatin A (TSA) (Sigma-Aldrich). Cells were harvested on day 4 for RNA and DNA extraction.

\section{Bisulfite-modification and methylation-specific PCR (MSP)}

Bisulfite conversion and MSP reaction conditions of in vitro derived DNA was performed as specified previously [81]. For used DAPK1 MSP primers and cycle conditions see Additional file 6.

\section{Pyrosequencing}

Pyrosequencing of $14 \mathrm{CpG}$ sites within the DAPK1 5'UTR region was performed by using the PyroMark PCR Kit (Qiagen) for initial fragment amplification. The PyroMark96 ID device and the PyroGoldSQA reagent Kit (Qiagen) were used as previously described [18]. The DAPK1 assay was designed by using the Pyromark Assay Design Software (Qiagen) and all primers are listed in Additional file 7 .

\section{GTPases pulldown}

Activation of both Rac1 and RhoA was measured by using the Active Rac1 Detection Kit (\#8815, Cell Signaling, Danvers, MA, USA) and the Active Rho Detection Kit (\#8820, Cell Signaling) respectively, according to the manufacturer's instructions. In brief, single-cell $\Delta \mathrm{pBK}$ ITIH5 and mock clones were cultured in G418 containing growth medium for $48 \mathrm{~h}$. Subsequent to the cell lysis, $550 \mu \mathrm{g}$ of total cell protein lysate for each clone was mixed with $20 \mu \mathrm{g}$ of GST-PAK1-PBD capturing (active) RAC1-GTP or GST-Rhotekin-RBD for RhoA. Glutathione matrix-immobilized Rac1-GTP or Rho-GTP was eluted in SDS sample buffer supplemented with DTT. After heat denaturation $\left(5 \mathrm{~min}, 95^{\circ} \mathrm{C}\right) \mathrm{Rac} 1$ and RhoA proteins were detected by western blot analysis using specific antibodies (see Additional file 8). Total cellular RAC1 or RhoA protein was determined for each sample and used for normalization.

\section{Western blot}

Western blot analysis was performed as previously described [82] but slightly modified as following: Proteins were extracted in RIPA lysis buffer, then separated in $4-12 \%$ Bis-Tris gels (Invitrogen Life Technologies, Darmstadt, Germany) under reducing (50 mM DTT) conditions using MES-SDS running buffer and electroblotted onto nitrocellulose membranes $(0.2 \mu \mathrm{m})$. Commercial primary antibodies used are listed in Additional file 8 . The generated anti-ITIH5 antibody was previously characterized [18]. Equal protein loading was monitored by using $\beta$-actin specific antibody.

\section{Immunofluorescence}

MDA-MB-231-ITIH5 $\triangle$ pBK-ITIH5 and mock clones $\left(3 \times 10^{4}\right.$ cells/well $)$ were plated onto $12 \mathrm{~mm}$ round glass coverslips. After $24 \mathrm{~h}$ incubation, cells were fixed with $4 \%$ paraformaldehyde (PFA) and $0.5 \%$ Triton X-100 in cytoskeleton buffer (10 mM PIPES, $150 \mathrm{mM} \mathrm{NaCl}, 5 \mathrm{mM}$ EGTA, $5 \mathrm{mM}$ glucose, and $5 \mathrm{mM} \mathrm{MgCl}_{2}, \mathrm{pH} 7.0$ ) for $10 \mathrm{~min}$ at room temperature. Afterwards, cells were gently washed twice with PBS and post-fixed with 4\% PFA for $10 \mathrm{~min}$ at room temperature. Subsequently, cells were washed thrice with cytoskeleton buffer. For vinculin labeling, cells were incubated with the monoclonal antibody hVIN-1 (Sigma-Aldrich, Deisenheim, Germany) for $30 \mathrm{~min}$ at room temperature followed by Alexa 488conjugated goat anti-mouse IgG (Molecular Probes, Eugene, OR). The actin cytoskeleton was labelled with Alexa 594-conjugated phalloidin (Molecular Probes). Coverslips were mounted in Prolong (Molecular Probes). Specimens were observed using an Axiovert 200 microscope (Zeiss, Jena, Germany) equipped with a PlanApochromat $100 \times / 1.40$ NA oil immersion objective in combination with $1.6 \times$ or $2.5 \times$ optovar optics. Images were recorded with a cooled, back-illuminated CCD camera (Cascade, Photometrics, Tucson, AZ) driven by IPLab Spectrum software (Scanalytics Inc., Rockville, MD).

\section{Scanning electron microscopy}

Cells were fixed in 3\% glutaraldehyde (in $0.1 \mathrm{M}$ Soerensen's phosphate buffer [13 mM NaH${ }_{2} \mathrm{PO}_{4} \times \mathrm{H}_{2} \mathrm{O}$; $\left.87 \mathrm{mM} \mathrm{Na} \mathrm{HPO}_{4} \times 2 \mathrm{H}_{2} \mathrm{O} ; \mathrm{pH} 7.4\right]$ ) for at least $1 \mathrm{~h}$, then rinsed in $0.1 \mathrm{M}$ Soerensen's phosphate buffer. Next, cells were dehydrated in a graded ethanol series $(30,50,70,90,3 \% \times 100 \%)$ and critical-point-dried in carbon dioxide (CPD 010, Balzers Union, FL). The dried samples were fixed on SEM stubs and sputtercoated with gold (SCD 030, Balzers Union), then analyzed with an ESEM XL 30 FEG (FEI Philips, Eindhoven, Netherlands) in high vacuum mode at an accelerating voltage of $10 \mathrm{kV}$. 


\section{Cell attachment assay}

Cell adhesion experiments were carried out as previously described [79] with minor modifications: Six-well plates were coated with HA (100 $\mu \mathrm{g} / \mathrm{ml}$; Sigma-Aldrich) or Matrigel $^{\mathrm{TM}}(10 \mu \mathrm{g} / \mathrm{ml}$; Sigma-Aldrich $)$ and cells $\left(5 \times 10^{5}\right.$ cells/well) were incubated to adhere on surface for $30 \mathrm{~min}$ at $37{ }^{\circ} \mathrm{C}$. Attached cells were fixed with $70 \%$ ethanol for $10 \mathrm{~min}$ and stained with $0.1 \%$ crystal violet. After 20 min cells were exhaustively washed with water and dried overnight. The dye was dissolved in $0.002 \%$ Triton X-100 in 100\% isopropanol and carried over into a 96-well plate to measure the optical density at $590 \mathrm{~nm}$ using an ELISA reader (SpectraMax 340; Molecular Devices; CA).

\section{Fabrication of silicone rubber substrates}

Substrate preparation and characterization of elastomer material properties (Young's modulus and Poisson's ratio) were performed as previously described [83]. In brief, cross-linked elastomeric silicone rubber was used (Sylgard 184, Dow Corning), which is supplied as a twocomponent kit consisting of base and cross-linker oil. Both components were mixed at a ratio of 1:50 and mixed with $5 \%(\mathrm{v} / \mathrm{v})$ yellow-green fluorescent nanobeads (0.2 $\mu \mathrm{m}$ diameter, FluoSpheres, Invitrogen). This prepolymer mixture was applied onto a micro-structured silicon dioxide mold containing $500 \mathrm{~nm}$ high microdots with an edge length of $2.5 \mu \mathrm{m}$ and a lattice constant of $3.5 \mu \mathrm{m}$, to generate a regular bead layer within the elastomeric substrate. The polymer layer was then covered by a glass coverslip. A defined layer thickness of $80 \mu \mathrm{m}$ was produced by putting spacers between the silicon surface and the coverslip. Pre-polymer mixtures were heat cross-linked $\left(60{ }^{\circ} \mathrm{C}\right)$ overnight and finally displayed a Poisson's ratio of 0.5 and a Young's modulus of $15 \mathrm{kPa}$. For cell culture, the silicon mold and spacer were removed and glass bottom covered elastomer substrates were glued to a $3.5 \mathrm{~cm}$ Petri dishes with $1.5 \mathrm{~cm}$ holes.

\section{Traction force microscopy and cell force retrieval}

Live cell analyses were performed at $37{ }^{\circ} \mathrm{C}$ and $5 \% \mathrm{CO}_{2}$ (cell incubator XL2, Carl Zeiss, Germany) using an inverted confocal laser scanning microscope (cLSM710, Carl Zeiss, Germany), utilizing a 40× EC Plan-Neofluar oil immersion objective (PH3, NA =1.3, Carl Zeiss, Germany). Images were taken using the imaging software ZEN 2.1, Carl Zeiss Germany). Confocal micrographs of the cells (phase contrast) and of yellow-green fluorescent beads were taken using an argon ion laser (488 nm) with a transmitted light detector and a 490$530 \mathrm{~nm}$ bandpass filter, respectively. Cells were seeded onto fibronectin-coated $\left(20 \mu \mathrm{g} / \mathrm{cm}^{2}\right)$ TFM substrates $48 \mathrm{~h}$ before measurement. Only well-adhered cells were analyzed. Traction forces applied by a single cell to an elastic substrate of defined stiffness cause deformations fields that were visualized by tracking fluorescent marker beads in the substrate. From the displacement of these particles cell forces were calculated. Substrate deformation was captured in the presence of cells and substrate relaxation was obtained after cell elimination by trypsinization. Cell area force fields (AFF) were retrieved from vector displacement fields (DVF) determined by correlating the nanobead displacement in the deformed and the relaxed, cell-free elastomer. MatLab-based algorithms were used for data processing as previously described $[29,84]$.

\section{XTT cell proliferation assay}

The XTT proliferation assay (Roche Diagnostics, Mannheim, Germany) was used and performed as previously described [16].

\section{Apoptosis assay}

Activity of the effector caspases 3 and 7 in ITIH5 and mock single-cell clones was analyzed by using the Apo-One ${ }^{\bullet}$ Homogeneous Caspase-3/7 Assay (Promega, Mannheim, Germany) according to the manufacturer's instructions. Briefly, cells $\left(1.5 \times 10^{4}\right)$ were seeded in 96cell culture wells and incubated overnight $\left(20 \% \mathrm{O}_{2}, 5 \%\right.$ $\left.\mathrm{CO}_{2}, 37{ }^{\circ} \mathrm{C}\right)$. Afterwards, staurosporine $(1 \mu \mathrm{M}$, SigmaAldrich, Deisenhofen, Germany) was applied to induce apoptosis. Fluorescence intensity was quantified by using an ELISA plate reader (excitation: $\lambda=485 \mathrm{~nm}$; emission: $\lambda=577 \mathrm{~nm})$.

\section{In vitro colony formation and migration studies}

Colony formation assays were performed as previously described [79]. In vitro motility was analyzed performing a monolayer scratch wound assay as previously specified [85].

\section{In vivo metastasis assay}

MDA-MB-231 cells $\left(3 \times 10^{6}\right)$ of the ITIH5 test set $(\Delta \mathrm{pBK}-\mathrm{ITIH} 5$ clones $)$ or the control set $(\Delta \mathrm{pBK}$-mock clones) were intravenously inoculated into the lateral tail vein of 7 week old female Balb/c $\mathrm{c}^{\text {nu/nu }}$ mice. After 50 days, mice were $\mu \mathrm{CT}$ scanned, and then sacrificed. Lungs were harvested, photographed with the Discovery V12 stereomicroscope (Zeiss), analyzed with DISKUS software package (Königswinter, Germany), formalin-fixed (10\%) and paraffin-embedded. H\&E-stained sections from each lung tissue as well as a further slide sectioned at $30 \mu \mathrm{m}$ increments in the vertical plane were examined by a pathologist in a blinded manner to quantify the number of micro-metastases. 


\section{In vivo micro-computed tomography}

Whole-body scans of mice were performed using noninvasive $\mu \mathrm{CT}$. A gantry-based dual energy microcomputed TomoScope 30s Duo (CT Imaging, Erlangen, Germany) was used. Matched pairs of mice ( $n=7$ each) were scanned 50 days after tumor cell injection and anaesthetized using a $1.5 \%$ isoflurane inhalation narcosis. Mice were scanned both natively and after intravenous application of eXIA ${ }^{\mathrm{sm}} 160$ (Binitio Biomedical, Ottawa, Canada), an iodine-based and radiopaque blood pool contrast agent. Injected dose of $0.1 \mathrm{ml} / 20 \mathrm{~g}$ body weight was used [86]. Images were reconstructed using a Feldkamp type reconstruction (CT-Imaging, Erlangen, Germany) generating a voxel size of $70 \times 70 \times 70 \mu \mathrm{m}^{3}$. Subsequently, images were analyzed using Amide [87]. 3D architecture was visualized using Imalytics Preclinical software [88].

\section{Gene expression profiling}

Gene expression profiling of the ITIH5 test set (three independent MDA-MB-231 $\triangle$ pBK-ITIH5 clones) and the control set (three independent MDA-MB-231 $\Delta \mathrm{pBK}$ mock clones) was carried out by the IZKF Chip-Facility (Interdisciplinary Centre for Clinical Research Aachen within the Medical faculty of the RWTH Aachen University) using the Affymetrix 1.0 ST gene array (Affymetrix, Santa Clara, CA).

Profiling of stably transfected MDA-MB-231 breast cancer cells was performed using BRB-ArrayTools developed by Dr. Richard Simon and BRB-ArrayTools Development Team version 4.3.0 - Beta. In order to identify the significantly regulated candidate genes the class comparison evaluation was used [89], which met the following criteria: Significantly $(p<0.05)$ differentially expressed with a minimal change in expression by 3 -fold. Exact permutation $p$ values for significant genes were computed based on 35 available permutations. Genes were excluded when less than $20 \%$ of expression data had at least a 1.5 -fold change in either direction from gene's median value. Gene Ontology (GO) categories were determined by applying a gene set comparison analysis that is similar to the gene set enrichment analysis described by Subramanian et al. [90]. Tests used to find significant gene sets were: LS/KS permutation test (to find gene sets which have more genes differentially expressed among the phenotype classes than expected by chance). Over-represented GO lists were considered significant when the threshold of determining significant gene sets is equal or below 0.005 (LS/KS permutation test).

\section{DNA methylation profiling}

DNA methylation profiles were analyzed in three independent MDA-MB-321 $\triangle$ pBK-ITIH5, two mock clones and WT by using the HumanMethylation450 Beadchip technology (Illumina, San Diego, USA). Hybridization of bisulfite converted DNA (200 ng) and initial data evaluation was performed by the DKFZ Gene Core Facility (Heidelberg, Germany).

Limma-T-test statistics was calculated in $R$ [91] to select for CpG sites with significant differences in DNA methylation (adjusted $p$ value $<0.05$ and 20\% differential DNA methylation level between both test groups). Cluster analysis of the CpG sites was performed with the "pheatmap package" for R using complete linkage and Euclidean distance [92]. The Gene Ontology analysis was performed using the GOrilla software tool to visualize GO terms of target (1511 GpG sites) and background list (all analyzed CpG sites) [93]. Overlap of significantly hyper- and hypomethylated CpG sites between $\triangle \mathrm{pBK}$-ITIH5 and $\triangle \mathrm{pBK}$ mock clones with gene set data bases was performed using a public gene set enrichment analysis platform (GSEA; http://www.broadinstitute.org/gsea/index.jsp) [90, 94]. The probes / CpG sites of the HumanMethylation450 BeadChip were furthermore annotated with previously published data on the presence of two histone H3 modifications (H3K4Me3 and H3K27Me3) close to a transcription start site in embryonic stem cells [39]. We used the information on the probed location (GRC36 reference) provided by the manufacturer (HumanMethylation450 v1.2 Manifest File). A promoter region that contained at least one probed CpG site with a significant difference in DNA methylation level was called deregulated (Additional file 3). The subsequent analysis was limited to the 12,564 (69\%) regions with a minimum of 5 probed CpG sites to reduce the bias introduced by a low coverage. Methylation $\beta$-values of multiple significant different methylated CpG sites were averaged after transformation to $\mathrm{M}$-values.

\section{Statistics}

Statistical analyses were performed using GraphPad Prism 5.0 (GraphPad Software Inc., La Jolla, CA) and SPSS 20.0 (SPSS, Chicago, IL). Differences were considered statistically significant if the two sided $p$-values were equal or below $5 \%(\leq 0.05)$. To compare two or more groups the Mann-Whitney or Kruskal-Wallis test was used, respectively. Overall survival (OS) was measured from surgery until death and was censored for patients alive at the last follow-up using the univariate log-rank tests.

\section{Additional files}

Additional file 1: Cell plasticity of ITIH5-expressing MDA-MB-231 single-cell clones. This figure shows morphological characteristics of independent MDA-MB-231 single-cell clones using phase-contrast microscopy. (DOCX 109 kb)

Additional file 2: Overlap of hypo-and hypermethylated CpG sites with published gene sets (GSEA). This table summarizes an overlap of significantly hyper- and hypomethylated CpG sites between $\triangle \mathrm{pBK}-\mathrm{ITIH} 5$ and $\triangle \mathrm{pBK}$-mock clones with gene set data bases. (XLS $72 \mathrm{~kb}$ ) 
Additional file 3: $\mathrm{CpG}$ sites with potential $\mathrm{H} 3$ marks. This table lists $\mathrm{CpG}$ sites of the HumanMethylation 450 BeadChip annotated with histone H3 modifications (H3K4Me3 and H3K27Me3). (XLSX $7866 \mathrm{~kb}$ )

Additional file 4: Identified promoter regions with potential $\mathrm{H3}$ marks that are hyper- or hypomethylated due to ITIH5 expression. This table illustrates the 242 different promoter regions featuring a significant association with a potential H3 methylation status described for ES cells, (XLSX $23 \mathrm{~kb})$

Additional file 5: Primer sequences and reaction conditions for RT-PCR experiments. This table illustrates the sequences of all primers used in this study for RT-PCR analysis. (XLS 28 kb)

Additional file 6: Primer sequences and PCR conditions for MSP analyses. This table illustrates the sequences of all primers used in this study for MSP analysis. (XLSX $10 \mathrm{~kb}$ )

Additional file 7: Primer sequences for pyrosequencing. This table shows sequences of DAPK1 pyrosequencing. (XLSX $10 \mathrm{~kb}$ )

Additional file 8: Primary antibodies used in this study. This table lists all primary andtiboides used in this study. (XLS $26 \mathrm{~kb}$ )

\section{Abbreviations}

2D: Two-dimensional; 3D: Three-dimensional; AFF: Area force field; ATCC: American Type Culture Collection; BM: Basement membrane; BP: Biological process; CA: California; CDNA: Copy number desoxyribonucleic acid; CMV: Cytomegalovirus; COL4: Collagen 4; CpG: 5'-Deoxycytidinephosphate-deoxyguanosine-3'; CSC: Cancer stem cell; DAPK1: Deathassociated protein kinase 1; DNA: Deoxyribonucleic acid; DNMT: DNA methyltransferase; dNTP: Desoxyribonucleosidtriphosphate; DSC2: Desmocollin 2; DSG2: Desmoglein 2; DSP: Desmoplakin; ECM: Extracellular matrix; EK: Ethics committee; EMT: Epithelial-tomesenchymal transition; ES: Embryonic stem cell; FA: Focal adhesion; FC: Fold change; FCS: Fetal calf serum; FFPE: Formalin fixed paraffin embedded; GAPDH: Glyeradehyde 3-phosphate dehydrogenase; GO: Gene ontology; GSEA: Gene set enrichment analysis; GTP: Hydrolyze guanosine triphosphate; h: Hour; H3K27me3: Trimethylation mark at K27; H3K4me3: Trimethylation mark at K4; HA: Hyaluronan; HABP: HA-binding protein; i.v: Intravenous; IL: Illinois; ITI: Inter-a-trypsin inhibitor; ITH: Inter-a-trypsin inhibitory heavy chain; IZKF: Interdisziplinäres Zentrum für Klinische Forschung; LANUV: Landesamt für Umwelt, Natur und Verbraucherschutz; MEF: Embryonic fibroblast; min: Minute; mRNA: Messenger ribo nucleic acid; MSP: Methylation specific PCR; n: Number; $n N$ : Nano Newton; NPC: Neural precursor cell; PBS: Phosphate-buffered saline; PCG: Polycomp-group; PCR: Polymerase chain reaction; ROI: Region of interest; RT: Room temperature; RT-PCR: Real-time PCR; RWTH: RheinischWestfälisch Technische Hochschule; s.e.m.: Standard error of the margin; siRNA: Small interfering RNA; TCGA: The Cancer Genome Atlas; TFM: Traction force microscopy; TGF: Transforming growth factor; trxG: Trithorax-group; TSS: Transcription start site; USA: United States of America; UTR: Untranslated region; VA: Virginia; VDF: Vector deformation field; VIT: Vault protein inter-atrypsin; vWA: Von Willebrand A; WI: Wisconsin; WT: Wildtype; $\mu C T$ : Microcomputed tomography

\section{Acknowledgement}

The excellent technical assistance of Roswitha Davtalab, Hiltrud Königs and Sonja von Serényi is thankfully acknowledged.

This paper is dedicated to the memory of our wonderful colleague Dr. Jürgen Veeck, who recently passed away in his personal fight against cancer.

\section{Funding}

This work was supported by the Fritz Thyssen Stiftung (Az.10.09.2.121) and by the ForSaTum grant from the European Union and NRW government (NRW-EU Ziel 2, (EFRE), 005-0908-0112) to the Institute for Laboratory Animal Science and the Department of Experimental Molecular Imaging.

\section{Availability of data and materials}

The datasets supporting the conclusions of this article are included within the article and its additional files.

The Affymetrix microarray data underlying gene expression analysis of this article are available at the European Bioinformatics Institute (EMBL-EBI) database (http://www.ebi.ac.uk) and assigned the identifier (E-MTAB-1813| http://www.ebi.ac.uk/arrayexpress| \#Login). HumanMethylation450 Beadchip data sets are accessible through accession number E-MTAB-5081 (http:// www.ebi.ac.uk/arrayexpress| \#Login).

\section{Authors' contributions}

MR participated in all experiments, in the design of the study and wrote the manuscript. VK, EN, RK and ED conceived and coordinated the study and edited the manuscript. EN, LG, JE, SKM, AGS, SH, ASS, RW, OK, WA, QL, WW, JV, JS involved in experimental data acquisition and analysis. TH and QL performed bioinformatics using R. FG developed imaging software. All authors read, critically revised, and approved the final manuscript.

\section{Competing interests}

Edgar Dahl and Michael Rose are cofounders of Qithera $\mathrm{GmbH}$, a company that seeks to develop novel anticancer compounds based on the ITIH5 tumor suppressor pathway.

\section{Consent for publication}

Not applicable.

\section{Ethics approval and consent to participate}

Not applicable.

\section{Author details}

'Institute of Pathology, Medical Faculty of the RWTH Aachen University, Aachen, Germany. ${ }^{2}$ Institute of Complex Systems, ICS-7: Biomechanics, Forschungszentrum Jülich $\mathrm{GmbH}$, Jülich, Germany. ${ }^{3}$ Department of Experimental Molecular Imaging (ExMl), Helmholtz Institute for Biomedical Engineering, Medical Faculty of the RWTH Aachen University, Aachen, Germany. ${ }^{4}$ Experimental Gene Therapy and Clinical Chemistry, Institute of Molecular Pathobiochemistry, Medical Faculty of the RWTH Aachen University, Aachen, Germany. ${ }^{5}$ Institute for Biomedical Engineering - Cell Biology, Medical Faculty of the RWTH Aachen University, Aachen, Germany. ${ }^{6} \mathrm{Helmholtz-Institute}$ for Biomedical Engineering-Stem Cell Biology and Cellular Engineering, Medical Faculty of the RWTH Aachen University, Aachen, Germany. ${ }^{7}$ Division of Medical Oncology, Department of Internal Medicine, Department of Pathology, GROW-School for Oncology and Developmental Biology, Maastricht University Medical Centre, Maastricht, The Netherlands. ${ }^{8}$ Institute for Laboratory Animal Science, Medical Faculty of the RWTH Aachen University, Aachen, Germany.

Received: 21 October 2016 Accepted: 30 January 2017 Published online: 23 February 2017

\section{References}

1. Bissell MJ, Radisky DC, Rizki A, Weaver VM, Petersen OW. The organizing principle: microenvironmental influences in the normal and malignant breast. Differentiation. 2002;70:537-46.

2. Le BJ, Xu R, Lee SY, Nelson CM, Rizki A, Alcaraz J, Bissell MJ. Cell shape regulates global histone acetylation in human mammary epithelial cells. Exp Cell Res. 2007:313:3066-75.

3. Zhuo L, Hascall VC, Kimata K. Inter-alpha-trypsin inhibitor, a covalent protein-glycosaminoglycan-protein complex. J Biol Chem. 2004;279: 38079-82.

4. Bost F, Diarra-Mehrpour M, Martin JP. Inter-alpha-trypsin inhibitor proteoglycan family - a group of proteins binding and stabilizing the extracellular matrix. Eur J Biochem. 1998:252:339-46.

5. Salier JP, Rouet P, Raguenez G, Daveau M. The inter-alpha-inhibitor family: from structure to regulation. Biochem J. 1996;315(Pt 1):1-9.

6. Huang L, Yoneda M, Kimata K. A serum-derived hyaluronan-associated protein (SHAP) is the heavy chain of the inter alpha-trypsin inhibitor. J Biol Chem. 1993:268:26725-30.

7. Ponta H, Sherman L, Herrlich PA. CD44: from adhesion molecules to signalling regulators. Nat Rev Mol Cell Biol. 2003:4:33-45.

8. Lopez JI, Camenisch TD, Stevens MV, Sands BJ, McDonald J, Schroeder JA. CD44 attenuates metastatic invasion during breast cancer progression. Cancer Res. 2005;65:6755-63.

9. Chen L, Mao SJ, Mclean LR, Powers RW, Larsen WJ. Proteins of the interalpha-trypsin inhibitor family stabilize the cumulus extracellular matrix 
through their direct binding with hyaluronic acid. J Biol Chem. 1994;269: 28282-7.

10. Bourguignon J, Borghi H, Sesboue R, Diarra-Mehrpour M, Bernaudin JF, Metayer J, Martin JP, Thiberville L. Immunohistochemical distribution of inter-alpha-trypsin inhibitor chains in normal and malignant human lung tissue. J Histochem Cytochem. 1999;47:1625-32.

11. Werbowetski-Ogilvie TE, Agar NY, Waldkircher De Oliveira RM, Faury D, Antel JP, Jabado N, Del Maestro RF. Isolation of a natural inhibitor of human malignant glial cell invasion: inter alpha-trypsin inhibitor heavy chain 2. Cancer Res. 2006;66:1464-72.

12. Paris S, Sesboue R, Delpech B, Chauzy C, Thiberville L, Martin JP, Frebourg T, Diarra-Mehrpour $\mathrm{M}$. Inhibition of tumor growth and metastatic spreading by overexpression of inter-alpha-trypsin inhibitor family chains. Int I Cancer. 2002;97:615-20

13. Himmelfarb M, Klopocki E, Grube S, Staub E, Klaman I, Hinzmann B, Kristiansen G, Rosenthal A, Durst M, Dahl E. ITIH5, a novel member of the inter-alpha-trypsin inhibitor heavy chain family is downregulated in breast cancer. Cancer Lett. 2004;204:69-77.

14. Huth S, Heise R, Vetter-Kauczok CS, Skazik C, Marquardt Y, Czaja K, Knuchel R, Merk HF, Dahl E, Baron JM. Inter-alpha-trypsin inhibitor heavy chain 5 (ITHH) is overexpressed in inflammatory skin diseases and affects epidermal morphology in constitutive knockout mice and murine 3D skin models. Exp Dermatol. 2015;24:363-86.

15. Anveden A, Sjoholm K, Jacobson P, Palsdottir V, Walley AJ, Froguel P, Al-Daghri N, Mcternan PG, Mejhert N, Arner P, et al. ITH -5 expression in human adipose tissue is increased in obesity. Obesity (Silver Spring). 2012;20:708-14.

16. Veeck J, Chorovicer M, Naami A, Breuer E, Zafrakas M, Bektas N, Durst M, Kristiansen G, Wild PJ, Hartmann A, et al. The extracellular matrix protein ITIH5 is a novel prognostic marker in invasive node-negative breast cancer and its aberrant expression is caused by promoter hypermethylation. Oncogene. 2008;27:865-76.

17. Hamm A, Veeck J, Bektas N, Wild PJ, Hartmann A, Heindrichs U, Kristiansen G, Werbowetski-Ogilvie T, Del Maestro R, Knuechel R, et al. Frequent expression loss of Inter-alpha-trypsin inhibitor heavy chain (ITIH) genes in multiple human solid tumors: a systematic expression analysis. BMC Cancer. 2008;8:25.

18. Rose M, Gaisa NT, Antony P, Fiedler D, Heidenreich A, Otto W, Denzinger S, Bertz S, Hartmann A, Karl A, et al. Epigenetic inactivation of ITIH5 promotes bladder cancer progression and predicts early relapse of pT1 high grade urothelial tumours. Carcinogenesis. 2013;35:727-36.

19. Kloten V, Rose M, Kaspar S, von Stillfried SS, Knuchel R, Dahl E. Epigenetic inactivation of the novel candidate tumor suppressor gene ITIH5 in colon cancer predicts unfavorable overall survival in the $\mathrm{CpG}$ island methylator phenotype. Epigenetics. 2014;9:1290-301.

20. Mai C, Zhao JJ, Tang XF, Wang W, Pan K, Pan QZ, Zhang XF, Jiang SS, Zhao BW, Li YF, et al. Decreased ITIH5 expression is associated with poor prognosis in primary gastric cancer. Med Oncol. 2014;31:53.

21. Dotsch MM, Kloten V, Schlensog M, Heide T, Braunschweig T, Veeck J, Petersen I, Knuchel R, Dahl E. Low expression of ITIH5 in adenocarcinoma of the lung is associated with unfavorable patients' outcome. Epigenetics. 2015;10:903-12.

22. Wu K, Zhang X, Li F, Xiao D, Hou Y, Zhu S, Liu D, Ye X, Ye M, Yang J, et al. Frequent alterations in cytoskeleton remodelling genes in primary and metastatic lung adenocarcinomas. Nat Commun. 2015;6:10131.

23. Veeck J, Breuer E, Rose M, Chorovicer M, Naami A, Bektas N, Alkaya S, Horn F, von Stillfried S, Hartmann A, et al. [Novel prognostic marker in invasive breast cancer. ITIH5 expression is abrogated by aberrant promoter methylation]. Pathologe. 2008;29 Suppl 2:338-46.

24. Ringner M, Fredlund E, Hakkinen J, Borg A, Staaf J. GOBO: gene expressionbased outcome for breast cancer online. Plos One. 2011;6:e17911.

25. The Cancer Genome Atlas Network. Comprehensive molecular portraits of human breast tumours. Nature. 2012;490:61-70.

26. Hu Z, Fan C, Oh DS, Marron JS, He X, Qaqish BF, Livasy C, Carey LA, Reynolds $E$, Dressler $L$, et al. The molecular portraits of breast tumors are conserved across microarray platforms. BMC Genomics. 2006;7:96.

27. Pelham Jr RJ, Wang YI. Cell locomotion and focal adhesions are regulated by substrate flexibility. Proc Natl Acad Sci U S A. 1997;94:13661-5.

28. Butcher DT, Alliston T, Weaver VM. A tense situation: forcing tumour progression. Nat Rev Cancer. 2009;9:108-22.

29. Merkel R, Kirchgessner N, Cesa CM, Hoffmann B. Cell force microscopy on elastic layers of finite thickness. Biophys J. 2007;93:3314-23.
30. Koch TM, Münster S, Bonakdar N, Butler JP, Fabry B. 3D traction forces in cancer cell invasion. Plos One. 2012;7:e33476.

31. Yu H, Mouw JK, Weaver VM. Forcing form and function: biomechanical regulation of tumor evolution. Trends Cell Biol. 2011;21:47-56.

32. Kostic $A$, Lynch $C D$, Sheetz MP. Differential matrix rigidity response in breast cancer cell lines correlates with the tissue tropism. Plos One. 2009;4:e6361.

33. Welf ES, Naik UP, Ogunnaike BA. Probabilistic modeling and analysis of the effects of extra-cellular matrix density on the sizes, shapes, and locations of integrin clusters in adherent cells. BMC Biophys. 2011;4:15.

34. Huveneers S, Danen EH. Adhesion signaling — crosstalk between integrins, Src and Rho. J Cell Sci. 2009;122:1059-69.

35. Mousavi SJ, Doweidar MH. Role of mechanical cues in cell differentiation and proliferation: a 3D numerical model. Plos One. 2015;10:e0124529.

36. Lelievre SA. Contributions of extracellular matrix signaling and tissue architecture to nuclear mechanisms and spatial organization of gene expression control. Biochim Biophys Acta. 2009;1790:925-35.

37. Nishino K, Toyoda M, Yamazaki-Inoue M, Fukawatase Y, Chikazawa E, Sakaguchi $\mathrm{H}$, Akutsu $\mathrm{H}$, Umezawa A. DNA methylation dynamics in human induced pluripotent stem cells over time. Plos Genet. 2011;7:e1002085.

38. Lister R, Pelizzola M, Kida YS, Hawkins RD, Nery JR, Hon G, AntosiewiczBourget J, O'Malley R, Castanon R, Klugman S, et al. Hotspots of aberrant epigenomic reprogramming in human induced pluripotent stem cells. Nature. 2011:471:68-73.

39. Ku M, Koche RP, Rheinbay E, Mendenhall EM, Endoh M, Mikkelsen TS, Presser A, Nusbaum C, Xie X, Chi AS, et al. Genomewide analysis of PRC1 and PRC2 occupancy identifies two classes of bivalent domains. Plos Genet. 2008;4:e1000242.

40. Li LC, Dahiya R. MethPrimer: designing primers for methylation PCRs. Bioinformatics. 2002;18:1427-31.

41. Cartharius K, Frech K, Grote K, Klocke B, Haltmeier M, Klingenhoff A, Frisch $M$, Bayerlein $M$, Werner T. MatInspector and beyond: promoter analysis based on transcription factor binding sites. Bioinformatics. 2005;21:2933-42.

42. Raveh T, Kimchi A. DAP kinase-a proapoptotic gene that functions as a tumor suppressor. Exp Cell Res. 2001;264:185-92.

43. Kuo JC, Lin JR, Staddon JM, Hosoya H, Chen RH. Uncoordinated regulation of stress fibers and focal adhesions by DAP kinase. J Cell Sci. 2003;116:4777-90.

44. Kuo JC, Wang WJ, Yao CC, Wu PR, Chen RH. The tumor suppressor DAPK inhibits cell motility by blocking the integrin-mediated polarity pathway. J Cell Biol. 2006:172:619-31.

45. Selbi W, de la Motte CA, Hascall VC, Day AJ, Bowen T, Phillips AO. Characterization of hyaluronan cable structure and function in renal proximal tubular epithelial cells. Kidney Int. 2006;70:1287-95.

46. Zhuo L, Kimata K. Structure and function of inter-alpha-trypsin inhibitor heavy chains. Connect Tissue Res. 2008;49:311-20.

47. Hood JD, Cheresh DA. Role of integrins in cell invasion and migration. Nat Rev Cancer. 2002;2:91-100.

48. Chaudhuri O, Koshy ST, Branco Da CC, Shin JW, Verbeke CS, Allison KH, Mooney DJ. Extracellular matrix stiffness and composition jointly regulate the induction of malignant phenotypes in mammary epithelium. Nat Mater. 2014;13:970-8.

49. Cluzel C, Saltel F, Lussi J, Paulhe F, Imhof BA, Wehrle-Haller B. The mechanisms and dynamics of (alpha) $\vee$ (beta) 3 integrin clustering in living cells. J Cell Biol. 2005;171:383-92.

50. Ramirez NE, Zhang Z, Madamanchi A, Boyd KL, O'Rear LD, Nashabi A, Li Z, Dupont WD, Zijlstra A, Zutter MM. The alpha (2) beta (1) integrin is a metastasis suppressor in mouse models and human cancer. J Clin Invest. 2011;121:226-37.

51. Madamanchi A, Zijlstra A, Zutter MM. Flipping the switch: integrin switching provides metastatic competence. Sci Signal. 2014;7:e9.

52. Rottner K, Stradal TE. Actin dynamics and turnover in cell motility. Curr Opin Cell Biol. 2011;23:569-78.

53. Danen EH, Van RJ, Franken W, Huveneers S, Sonneveld P, Jalink K, Sonnenberg A. Integrins control motile strategy through a Rho-cofilin pathway. J Cell Biol. 2005;169:515-26.

54. White DP, Caswell PT, Norman JC. alpha v beta3 and alpha5beta1 integrin recycling pathways dictate downstream Rho kinase signaling to regulate persistent cell migration. J Cell Biol. 2007;177:515-25.

55. Shiu YT, Li S, Marganski WA, Usami S, Schwartz MA, Wang YI, Dembo M, Chien S. Rho mediates the shear-enhancement of endothelial cell migration and traction force generation. Biophys J. 2004;86:2558-65. 
56. Truong HH, Xiong J, Ghotra VP, Nirmala E, Haazen L, Le Devedec SE, Balcioglu $\mathrm{HE}$, He S, Snaar-Jagalska BE, Vreugdenhil E, et al. beta1 integrin inhibition elicits a prometastatic switch through the TGFbeta-miR-200-ZEB network in Ecadherin-positive triple-negative breast cancer. Sci Signal. 2014;7:ra15.

57. Wu J, Xu X, Lee EJ, Shull AY, Pei L, Awan F, Wang X, Choi JH, Deng L, Xin $\mathrm{HB}$, et al.: Phenotypic alteration of CD8+ T cells in chronic lymphocytic leukemia is associated with epigenetic reprogramming. Oncotarget. 2016. doi:10.18632/oncotarget.9941.

58. Singovski G, Bernal C, Kuciak M, Siegl-Cachedenier I, Conod A, Ruiz IA. In vivo epigenetic reprogramming of primary human colon cancer cells enhances metastases. J Mol Cell Biol. 2016;8:157-73.

59. Harikumar A, Meshorer E. Chromatin remodeling and bivalent histone modifications in embryonic stem cells. EMBO Rep. 2015;16:1609-19.

60. Lin B, Lee H, Yoon JG, Madan A, Wayner E, Tonning S, Hothi P, Schroeder B, Ulasov I, Foltz G, et al. Global analysis of H3K4me3 and H3K27me3 profiles in glioblastoma stem cells and identification of SLC17A7 as a bivalent tumor suppressor gene. Oncotarget. 2015;6:5369-81.

61. Easwaran H, Johnstone SE, Van NL, Ohm J, Mosbruger T, Wang Q, Aryee MJ, Joyce $\mathrm{P}$, Ahuja N, Weisenberger $\mathrm{D}$, et al. A DNA hypermethylation module for the stem/progenitor cell signature of cancer. Genome Res. 2012;22:837-49.

62. Gal-Yam EN, Egger G, Iniguez L, Holster H, Einarsson S, Zhang X, Lin JC, Liang G, Jones PA, Tanay A. Frequent switching of polycomb repressive marks and DNA hypermethylation in the PC3 prostate cancer cell line. Proc Natl Acad Sci U S A. 2008;105:12979-84.

63. Schlesinger $Y$, Straussman $R$, Keshet I, Farkash S, Hecht M, Zimmerman J, Eden E, Yakhini Z, Ben-Shushan E, Reubinoff BE, et al. Polycomb-mediated methylation on Lys 27 of histone $\mathrm{H} 3$ pre-marks genes for de novo methylation in cancer. Nat Genet. 2007:39:232-6.

64. Vire E, Brenner C, Deplus R, Blanchon L, Fraga M, Didelot C, Morey L, Van EA, Bernard D, Vanderwinden JM, et al. The polycomb group protein EZH2 directly controls DNA methylation. Nature. 2006;439:871-4.

65. Deiss LP, Feinstein $\mathrm{E}$, Berissi $\mathrm{H}$, Cohen $\mathrm{O}$, Kimchi A. Identification of a novel serine/threonine kinase and a novel $15-\mathrm{kD}$ protein as potential mediators of the gamma interferon-induced cell death. Genes Dev. 1995;9:15-30

66. Cohen O, Feinstein E, Kimchi A. DAP-kinase is a Ca2+/calmodulindependent, cytoskeletal-associated protein kinase, with cell death-inducing functions that depend on its catalytic activity. EMBO J. 1997;16:998-1008.

67. Yamamoto M, Hioki T, Ishii T, Nakajima-lijima S, Uchino S. DAP kinase activity is critical for C (2)-ceramide-induced apoptosis in PC12 cells. Eur J Biochem. 2002;269:139-47.

68. Wang WJ, Kuo JC, Yao CC, Chen RH. DAP-kinase induces apoptosis by suppressing integrin activity and disrupting matrix survival signals. J Cell Biol. 2002;159:169-79.

69. Sheridan C, Kishimoto H, Fuchs RK, Mehrotra S, Bhat-Nakshatri P, Turner CH, Goulet Jr R, Badve S, Nakshatri H. CD44+/CD24- breast cancer cells exhibit enhanced invasive properties: an early step necessary for metastasis. Breast Cancer Res. 2006:8:R59.

70. Blick T, Hugo H, Widodo E, Waltham M, Pinto C, Mani SA, Weinberg RA, Neve RM, Lenburg ME, Thompson EW. Epithelial mesenchymal transition traits in human breast cancer cell lines parallel the CD44 (hi/) CD24 (lo/-) stem cell phenotype in human breast cancer. J Mammary Gland Biol Neoplasia. 2010;15:235-52.

71. Bissell MJ, Hall HG, Parry G. How does the extracellular matrix direct gene expression? J Theor Biol. 1982;99:31-68.

72. Sandal T, Valyi-Nagy K, Spencer VA, Folberg R, Bissell MJ, Maniotis AJ. Epigenetic reversion of breast carcinoma phenotype is accompanied by changes in DNA sequestration as measured by Alul restriction enzyme. Am J Pathol. 2007;170:1739-49.

73. Swift J, Discher DE. The nuclear lamina is mechano-responsive to ECM elasticity in mature tissue. J Cell Sci. 2014;127:3005-15.

74. Maya-Mendoza A, Bartek J, Jackson DA, Streuli CH. Cellular microenvironment controls the nuclear architecture of breast epithelia through beta1-integrin. Cell Cycle. 2016;15:345-56.

75. Charafe-Jauffret $E$, Ginestier $C$, lovino F, Wicinski J, Cervera N, Finetti $P$, Hur MH, Diebel ME, Monville F, Dutcher J, et al. Breast cancer cell lines contain functional cancer stem cells with metastatic capacity and a distinct molecular signature. Cancer Res. 2009;69:1302-13.

76. Jing H, Liaw L, Friesel R, Vary C, Hua S, Yang X. Suppression of Spry4 enhances cancer stem cell properties of human MDA-MB-231 breast carcinoma cells. Cancer Cell Int. 2016:16. doi:10.1186/s12935-016-0292-7.

77. ATCC Bulletin: Maintaining high standards in cell culture. Manassas: American Type Culture Collection; 2010. https://www.atcc.org/ /media/ PDFs/CellBiologyStandards.ashx.
78. Veeck J, Niederacher D, An H, Klopocki E, Wiesmann F, Betz B, Galm O, Camara O, Durst M, Kristiansen $\mathrm{G}$, et al. Aberrant methylation of the Wnt antagonist SFRP1 in breast cancer is associated with unfavourable prognosis. Oncogene. 2006;25:3479-88.

79. Noetzel E, Rose M, Bornemann J, Gajewski M, Knuchel R, Dahl E. Nuclear transport receptor karyopherin-alpha2 promotes malignant breast cancer phenotypes in vitro. Oncogene. 2011;31:2101-14.

80. Noetzel E, Rose M, Sevinc E, Hilgers RD, Hartmann A, Naami A, Knuchel R, Dahl E. Intermediate filament dynamics and breast cancer: aberrant promoter methylation of the synemin gene is associated with early tumor relapse. Oncogene. 2010;29:4814-25.

81. Noetzel E, Veeck J, Niederacher D, Galm O, Horn F, Hartmann A, Knuchel R, Dahl E. Promoter methylation-associated loss of ID4 expression is a marker of tumour recurrence in human breast cancer. BMC Cancer. 2008;8:154.

82. Meurer SK, Alsamman M, Sahin H, Wasmuth HE, Kisseleva T, Brenner DA, Trautwein C, Weiskirchen R, Scholten D. Overexpression of endoglin modulates TGF-beta1-signalling pathways in a novel immortalized mouse hepatic stellate cell line. Plos One. 2013;8:e56116.

83. Cesa CM, Kirchgessner N, Mayer D, Schwarz US, Hoffmann B, Merkel R. Micropatterned silicone elastomer substrates for high resolution analysis of cellular force patterns. Rev Sci Instrum. 2007;78:034301.

84. Hersch N, Wolters B, Dreissen G, Springer R, Kirchgeßner N, Merkel R, Hoffmann B. The constant beat: cardiomyocytes adapt their forces by equal contraction upon environmental stiffening. Biol Open. 2013;2:351-61.

85. Kristiansen G, Hu J, Wichmann D, Stiehl DP, Rose M, Gerhardt J, Bohnert A, Ten HA, Moch H, Raleigh J, et al. Endogenous myoglobin in breast cancer is hypoxia-inducible by alternative transcription and functions to impair mitochondrial activity: a role in tumor suppression? J Biol Chem. 2011;286: 43417-28.

86. Willekens I, Lahoutte T, Buls N, Vanhove C, Deklerck R, Bossuyt A, de Mey J. Time-course of contrast enhancement in spleen and liver with exia 160, fenestra LC, and VC. Mol Imaging Biol. 2009;11:128-35.

87. Loening AM, Gambhir SS. AMIDE: a free software tool for multimodality medical image analysis. Mol Imaging. 2003;2:131-7.

88. Gremse F, Stärk M, Ehling J, Menzel JR, Lammers T, Kiessling F. Imalytics preclinical: interactive analysis of biomedical volume data. Theranostics. 2016;6:328-41.

89. Simon R, Lam A, Li MC, Ngan M, Menenzes S, Zhao Y. Analysis of gene expression data using BRB-ArrayTools. Cancer Informat. 2007;3:11-7.

90. Subramanian A, Tamayo P, Mootha VK, Mukherjee S, Ebert BL, Gillette MA, Paulovich A, Pomeroy SL, Golub TR, Lander ES, et al. Gene set enrichment analysis: a knowledge-based approach for interpreting genome-wide expression profiles. Proc Natl Acad Sci U S A. 2005;102:15545-50.

91. R Core Team: R: A language and environment for statistical computing. Vienna: R Foundation for Statistical Computing; 2016. http://www.R-project.org/.

92. Raivo Kolde: pheatmap: Pretty Heatmaps. R package version 1.0.8. 2015. https://cran.r-project.org/web/packages/pheatmap/index.html.

93. Eden E, Navon R, Steinfeld I, Lipson D, Yakhini Z. GOrilla: a tool for discovery and visualization of enriched $\mathrm{GO}$ terms in ranked gene lists. BMC Bioinformatics. 2009;10:48.

94. Mootha VK, Lindgren CM, Eriksson KF, Subramanian A, Sihag S, Lehar J, Puigserver P, Carlsson E, Ridderstrale M, Laurila E, et al. PGC-1alpharesponsive genes involved in oxidative phosphorylation are coordinately downregulated in human diabetes. Nat Genet. 2003;34:267-73.

\section{Submit your next manuscript to BioMed Central and we will help you at every step:}

- We accept pre-submission inquiries

- Our selector tool helps you to find the most relevant journal

- We provide round the clock customer support

- Convenient online submission

- Thorough peer review

- Inclusion in PubMed and all major indexing services

- Maximum visibility for your research

Submit your manuscript at www.biomedcentral.com/submit 